Article

\title{
Towards Distributed Recycling with Additive Manufacturing of PET Flake Feedstocks
}

\author{
Helen A. Little ${ }^{1}$, Nagendra G. Tanikella ${ }^{2}$, Matthew J. Reich ${ }^{2}$, Matthew J. Fiedler ${ }^{1}$, \\ Samantha L. Snabes ${ }^{1}$ and Joshua M. Pearce $2,3,4, * \mathbb{D}$ \\ 1 re:3D Inc., 1100 Hercules STE 220, Houston, TX 77058, USA; helen@re3d.org (H.A.L.); \\ matthew@re3d.org (M.J.F.); samantha@re3d.org (S.L.S.) \\ 2 Department of Material Science and Engineering, Michigan Technological University, \\ Houghton, MI 49931, USA; ngtanike@mtu.edu (N.G.T.); mjreich@mtu.edu (M.J.R.) \\ 3 Department of Electrical and Computer Engineering, Michigan Technological University, \\ Houghton, MI 49931, USA \\ 4 Department of Electronics and Nanoengineering, School of Electrical Engineering, Aalto University, \\ 00076 Espoo, Finland \\ * Correspondence: pearce@mtu.edu; Tel.: +1-906-487-1466
}

Received: 28 July 2020; Accepted: 22 September 2020; Published: 25 September 2020

check for updates

\begin{abstract}
This study explores the potential to reach a circular economy for post-consumer Recycled Polyethylene Terephthalate (rPET) packaging and bottles by using it as a Distributed Recycling for Additive Manufacturing (DRAM) feedstock. Specifically, for the first time, rPET water bottle flake is processed using only an open source toolchain with Fused Particle Fabrication (FPF) or Fused Granular Fabrication (FGF) processing rather than first converting it to filament. In this study, first the impact of granulation, sifting, and heating (and their sequential combination) is quantified on the shape and size distribution of the rPET flakes. Then 3D printing tests were performed on the rPET flake with two different feed systems: an external feeder and feed tube augmented with a motorized auger screw, and an extruder-mounted hopper that enables direct 3D printing. Two Gigabot $X$ machines were used, each with the different feed systems, and one without and the latter with extended part cooling. 3D print settings were optimized based on thermal characterization, and both systems were shown to 3D print rPET directly from shredded water bottles. Mechanical testing showed the importance of isolating rPET from moisture and that geometry was important for uniform extrusion. The mechanical strength of 3D-printed parts with FPF and inconsistent flow is lower than optimized fused filament, but adequate for a wide range of applications. Future work is needed to improve consistency and enable water bottles to be used as a widespread DRAM feedstock.
\end{abstract}

Keywords: polymers; recycling; waste plastic; upcycle; circular economy; PET; additive manufacturing; distributed recycling; distributed manufacturing; 3D printing

\section{Introduction}

The vast majority of plastics end up landfilled or contaminating the natural environment, as the global polymer recycling rate is an embarrassingly low $9 \%$ [1]. The problems of plastic recycling were recently highlighted when China imposed an import ban on waste plastic [2], which stalled global recycling efforts [3-5]. Without China, large-scale centralized plastic recycling has become uneconomic in many cases, and many municipalities have stopped recycling [6]. Part of the problem is that it is costly to separate the numerous types of plastic, and as consumers have no direct financial incentive to do it in conventional centralized recycling, increasingly sophisticated sorting technologies are proposed [7] to reach a circular economy [8-10]. 
Another approach to reach a circular economy for plastic is Distributed Recycling for Additive Manufacturing (DRAM) [11-13]. In the DRAM methodology, consumers have an economic incentive $[11,13]$ to recycle because they can use their waste as feedstock for a wide range of consumer products that can be produced for a fraction of conventional costs of equivalent products [14-17]. DRAM is a new technology that has the potential to radically impact global value chains [18]. Early DRAM work was centered on open source waste plastic extruders known as recyclebots, which upcycled post-consumer plastic waste into 3D printing filament $[19,20]$. In addition to reducing 3D printing costs by several orders of magnitude, it decreased embodied energy of 3D printing filament by $90 \%$ [21-23]. The open source 3D printing community, having evolved from the Self-Replicating Rapid Prototyper (RepRap) model [24-26], have embraced open source methods to recycle 3D printing waste [27] particularly for the two most popular fused filament materials: Polylactic Acid (PLA) [28-31] and Acrylonitrile Butadiene Styrene (ABS) [11,32-35]. More common thermoplastics were more challenging but have been successfully converted to filament including High-Density Polyethylene (HDPE) [19,36,37], Polypropylene (PP) and Polystyrene (PS) [37], Thermoplastic Polyurethane (TPU) [38], Linear Low-Density Polyethylene (LLDPE) and Low-Density Polyethylene (LDPE) [39], and Polycarbonate (PC) [40]. However, each melt and extrude cycle of a recyclebot impairs the mechanical properties of PLA [29], HDPE [41], and even of Polyethylene Terephthalate (PET) [42]. This limits the recycling cycles to approximately five [29] before reinforcement or blending with virgin materials becomes necessary. Polymer composites using carbon-reinforced plastic [43], fiber-filled composites [44,45], and various types of waste wood [46,47] have been used in recyclebot systems, and more complex DRAM systems can use 3D-printed PC as molds for intrusion molding [40] for windshield wiper composites [48] as well as Acrylonitrile Styrene Acrylate (ASA) and stamp sand waste composites [49]. Zander et al. [50] has studied PET, PP, and PS blends with Styrene Ethylene Butylene Styrene (SEBS) and maleic anhydride compatibilizers that were able to increase tensile strength from 19 to $23 \mathrm{MPa}$, although pure recycled PET had the highest tensile strength of $35 \mathrm{MPa}$.

This is part of the reason that the holy grail of DRAM has been PET. Although PET is only the 6th most commonly produced plastic, it is one of the most easily identifiable polymer waste streams for consumers [1] and is already widely recycled through centralized processes [51]. Although, the majority of centralized recycling is downcycling [52], it is, as noted earlier, not nearly at the rate to drive a circular economy. PET is an excellent water and moisture barrier, so it is used extensively in the packaging industry for consumable packaging of water, soft drinks, and foods [53]. PET use is expected to maintain a growth rate of $4.5 \% /$ year [54]. PET water bottles are easy to envision recycling at home as they are already clean and they are available in such large quantities globally as more than a million plastic bottles are produced every minute [55]. A few companies sell PET filament for 3D printing including Verbatim, MadeSolid, and Ultrafuse, and a few others sell recycled PET filament including Refil and B-PET. Although recycled PET and PETG filament is available commercially [56,57], some companies have stopped production [58,59]. PET is less popular than PLA, ABS, and PETG (glycol-modified version of PET) because the $3 \mathrm{D}$ printing process is more challenging as PET has shrinkage and warpage issues from high fusion temperature and lack of control of crystallinity, water absorption (leading to molecular weight reduction), and weak interfacial welding between layers [60]. In the past, PET industrial waste has been shown to successfully 3D print with Fused Particle Fabrication (FPF) or Fused Granular Fabrication (FGF) 3D printers that fabricate products directly from chips [61].

In centralized recycling, contamination and moisture are the major causes of deterioration of both the physical and chemical properties of PET [51]. In fact, Awaja and Patel state that to make a food-grade PET, the recycled PET must be hydrolyzed, purified, and re-polymerized [51]. Packaging-grade PET has been thought to require an increase in the viscosity or decrease in the melt flow index for effective use in material extrusion AM. One way to approach this is to use pyromellitic dianhydride chain extenders to increase the melt flow index of rPET via reactive extrusion [62]. There have been sporadic claims 
of PET recycling in the maker community, but they have failed to gain traction the way PLA or ABS recycling has caught on-primarily due to challenges in reproducibility. Part of these challenges also stem from the differences in the water bottles themselves, which are under constant flux. For example, since 2000, the average weight of a 16.9 ounce PET plastic bottle has declined by nearly half to $9.89 \mathrm{~g}$, saving billions of pounds of PET resin [63]. The largest problem, however, has been identified as PET undergoes hydrolytic degradation during melt processing, resulting in reduced molecular weights and, if the feedstock is too wet, even total disintegration of the polymer. Fortunately, there has been progress, as Tech4Trade and other partners developed a complex custom recyclebot (the Thunderhead) specifically for PET recycling $[64,65]$. This system not only has a complex number and sophisticated heating zones, it also has a heated hopper to ensure that the feedstock flakes are always dry. The first systematic study of PET-based DRAM was conducted by Zander et al. in 2018 [60]. They found that the chemistry for different PET feedstocks was identical, and their rheological results showed that drying of the PET led to an increase in the viscosity [60]. Moreover, by altering the processing parameters, they were able to control crystallinity (24.9\% for no active cooling down to $12.2 \%$ for the water-cooled filament) [60]. Finally, Zander et al.'s results of a PET tensile strength of $35.1 \pm 8 \mathrm{MPa}$ was found to be extremely promising as a material for DRAM [60].

To build on these promising results, this study explores the potential of PET packaging as a DRAM feedstock further by using only an open source toolchain with FPF/FGF processing. Earlier work showed that rPET pellets were processible [61], and here the more challenging flakes from rPET water bottles are investigated as a direct feedstock for FPF/FGF processing, without intermediary processing steps to convert the flake to pellets or filament, and without adding other materials to create composites. In this study, first the impact of granulation, sifting, and heating is quantified on the shape and size distribution of the rPET flakes. Then a feeding study was performed to determine whether they could be 3D printed through a feed tube connected to an externally mounted hopper, or whether the flake needed to be direct 3D printed with a gravity-fed hopper mounted to the print head. Two Gigabot $\mathrm{X}$ machines were used: one with extended part cooling and one without. 3D print settings were optimized based on DSC testing for the latter, and mechanical testing was performed on 3D-printed tensile bars. Both types of Gigabot X printers were used to fabricate example products from rPET pellets to show potential use cases for rPET 3D prints. The results are presented and discussed in the context of future work to make water bottles a DRAM feedstock.

\section{Materials and Methods}

\subsection{Materials}

Two recycled PET (rPET) materials were tested (see Figure 1). First, Ultrafuse PET pellets, which have previously been shown to be conducive to FPF/FGF processing with a Gigabot $X$ prototype [61] with two hot zones were evaluated. This commercial recycled rPET was shown to have ideal temperature settings of 220 and $230^{\circ} \mathrm{C}$ for zone 1 and 2 respectfully. The print bed was set at $100^{\circ} \mathrm{C}$ and printing speeds from 5 to $30 \mathrm{~mm} / \mathrm{s}$ were all shown to be adequate [61]. The second material was granulated water bottles. Water bottles were collected in Houston, Texas, and consisted primarily of the brands Hill Country Fare $(0.11 \mathrm{~mm}$ thickness), Great Value $(0.092 \mathrm{~mm})$, Ozarka $(0.09 \mathrm{~mm})$, and Texas Music Water $(0.09 \mathrm{~mm})$. Thickness measurements were taken using calipers at the top domed section of the bottles, where no seams were present. To convert PET water bottles into 3D-printable regrind material, the labels, caps, and adhesives were removed before granulating the bottles in a SHINI USA open rotor scissor cut granulator [66]. The granulator produced regrind small enough to pass through its grate, which has $5.84 \mathrm{~mm}$ diameter holes. After granulation, the regrind was dried in a food dehydrator for $24 \mathrm{~h}$ at $38^{\circ} \mathrm{C}$. This temperature was chosen to ensure that the rPET was not degraded by the drying process. In Figure 1, the pellets (blue) are not only more uniform but also bulkier than the granulated water bottle rPET (clear granulate). 


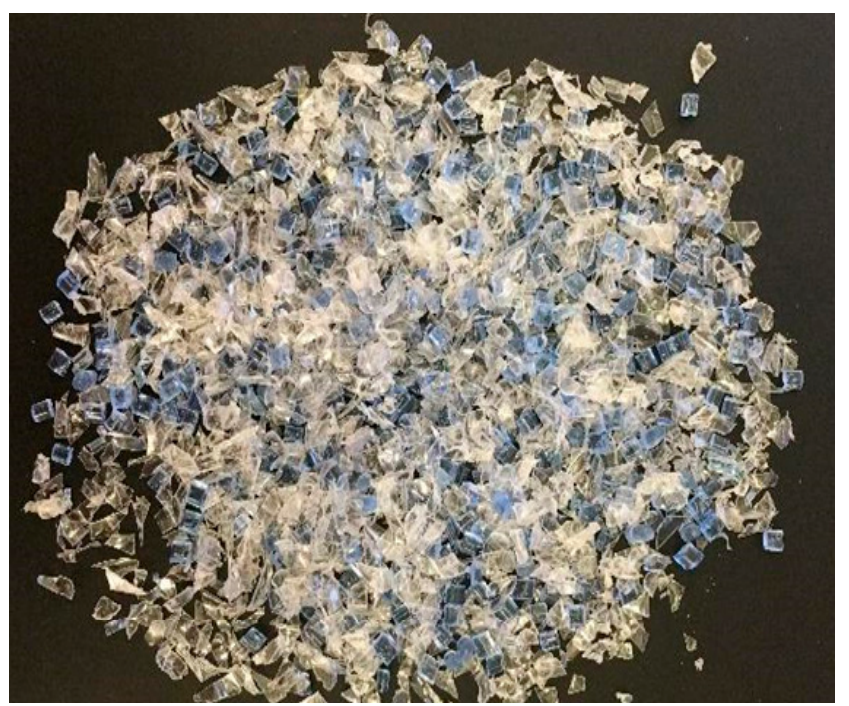

Figure 1. A 50:50 mix of Ultrafuse (blue pellets) and shredded water bottle (clear) to show relative, size, shape, and texture.

\subsection{Granulate Particle Analysis}

To compare the different PET sources, the granulate were characterized using FIJI ImageJ software [67]. The size characteristics of the particles for each starting material were quantified using digital imaging, and the open source Fiji/ImageJ Circularity, c, was defined as [68]:

$$
c=4 \pi \times \frac{A}{P^{2}}
$$

where $A$ is area in $\mathrm{mm}$ and $p$ is perimeter in $\mathrm{mm}$. Thus, a circularity value of 1.0 indicates a perfect circle; whereas as the values approach 0 , it indicates an increasingly elongated polygon. Then to compare the different processing methods for the PET water bottle granulate, the cross-sectional areas of granulate particles were plotted as normal distributions and compared.

\section{3. rPET Thermal Materials Characterization}

The thermal properties of rPET flake were first characterized with Differential Scanning Calorimetry (DSC) in order to have a starting point for the 3D printing process parameter optimization. Untreated rPET flake samples from post-consumer water bottles that were scanned for the DSC were then used as described in Sections 2.4 and 2.5. The rPET flakes were tested three times using the Netzsch DSC 404 furnace under pure argon flow of $50 \mathrm{~mL} / \mathrm{min}$ and a heating rate of $10^{\circ} \mathrm{C} / \mathrm{min}$. Background scans were performed on an empty aluminum crucible for each sample which generated calibration curves used to normalize the scan. The sample masses were measured with a precision of $\pm 0.01 \mathrm{mg}$ on a Sartorius scale and then entered into the Netzsch software. During the tests, each PET sample was placed into the aluminum crucible pan alongside an empty reference pan, and then the furnace chamber was purged and backfilled with argon to ensure no oxygen was present. Following this, the instrument heated the pans starting at $30.0^{\circ} \mathrm{C} \pm 7.5^{\circ} \mathrm{C}$. Heating at a constant rate of $10^{\circ} \mathrm{C}$ per minute, the crucibles were brought to a temperature of $300^{\circ} \mathrm{C}$ and then cooled back to room temperature.

\subsection{FPF/FGF 3D Printing}

Two approaches were taken to 3D print with flaked water bottles directly without first converting the rPET into filament. In the first approach a feed tube arrangement (Figure 2a) was used with a 3-heat-zone Gigabot $X$ (re:3D, Houston, TX, USA) (Figure 2b). The Gigabot $X$ is a direct pellet material extrusion-based 3D printer, with the nozzle arranged vertically as in Figure 2b. A compression screw and three hot zones enable a relatively constant flow of recycled material through the print nozzle. 
The turning of the compression screw acts the same as the main feed motor for a fused filament type RepRap machine. To assess the ability for a material to 3D print on Gigabot X design (Figure 2a), consistent flow through the 3D printer's feeding system was evaluated. Therefore, all samples of processed granulate were subjected to feed tests to identify which samples flowed through both the feed tube and feed tube adapter. A testing device was built with these components (Figure 2c). To perform the feed test on a material sample, the following steps were used:

1. Blocked bottom end of the feed tube.

2. Loaded the feed tube from the top with test material until it is full.

3. Unblocked the bottom of the feed tube to allow material to flow through via gravity.

4. Recorded whether all the material flowed through or became stuck inside the tube.

5. Repeated with the feed tube adapter attached at bottom of the feed tube to measure material flow through both the tube and adapter by massing the material as function of time.

To determine the 3D printing temperatures and test the extrusion rate of PET flake, the extruder was first flushed with Ultrafuse recycled PET (rPET) commercial pellets [69]. The PET water bottle flake was then fed directly into the feed throat to eliminate any effect of inconsistent granulate flow through the feed tube (Figure 2c). Initial extruder temperatures were set to $250{ }^{\circ} \mathrm{C}$ for the bottom heating zone closest to the nozzle, $240{ }^{\circ} \mathrm{C}$ for the middle, and $180^{\circ} \mathrm{C}$ for the top. The bottom zone temperature was set by incrementing up at $5{ }^{\circ} \mathrm{C}$ intervals until the granulate at the nozzle began to flow, and the top temperature was set low enough for the granulate at the top of the pellet screw to remain unmelted and provide pressure to extrude the melted plastic lower in the screw.

To flush the Ultrafuse rPET pellets out of the extruder and transition to extruding the rPET granulate, the extruder motor was rotated in increments of $200 \mathrm{~mm}$, at $600 \mathrm{steps} / \mathrm{mm}$ and at a speed of $3 \mathrm{~mm} / \mathrm{s}$. The use of millimeters in both the 3D printer firmware and in Simplify3D is designed for filament 3D printing, and describes the length of filament pulled by the motor and extruded. When open source firmware and slicing are developed specifically for direct-drive FPF, these values can be converted to rotations per minute using the steps per revolution for the motor to be consistent with what is occurring physically. When flushing from rPET pellets to PET water bottle granulate, the granulate did not extrude reliably. When flushing from water bottle granulate to rPET pellets, consistent extrusion was achieved. This indicated feeding issues due to the physical particle characteristics.

Therefore, additional processing methods were explored to decrease particle size and increase particle sphericity. This is because it is well known that spherical particles flow most easily [70], and although the impact of size on flow of particles is complex, in this system the smaller the particle size would have a lower probability of becoming jammed and restricting flow in the feeding tube. The processing methods explored to improve feeding and printability include:

1. Granulating Twice: Feeding granulated water bottles back into the SHINI granulator [66].

2. Sifting: Sifting through a 3D-printed sifter [71] with holes $5 \mathrm{~mm}$ in diameter and $2 \mathrm{~mm}$ deep. Sifting removes $40 \%$ of the granulate by weight, producing a $60 \%$ yield.

3. Heating:
a. Heating in a food dehydrator at $65.5^{\circ} \mathrm{C}$ for $24 \mathrm{~h}$.
b. Heating in an Analog Air Forced Analog Lab Oven (Quincy Lab) at $100^{\circ} \mathrm{C}$ for $1 \mathrm{~h}$.

4. Sequential sifting (2) and heating (3b): Sifted through the $5 \mathrm{~mm}$ hole sifter, then heated in the oven at $100{ }^{\circ} \mathrm{C}$ for one hour.

Additional tests were conducted to further quantify factors affecting particle shape when heated. Fiji/ImageJ measures a curled particle as having a smaller cross-sectional area than if the same particle were flattened. To better measure particle area changes without the factor of curling, flat $25.4 \times 25.4 \mathrm{~mm}$ square samples $\left(6.45 \mathrm{~cm}^{2}\right)$ were cut from the top portion of water bottles and submitted to various 
heating tests. To evaluate the diversity of plastic PET previously reported, five different brands of water bottles were assessed: Baraka, Hill Country Fair, Great Value, Ozarka, and Texas Music Water. Baraka bottles were sourced from a U.S. Air Force Forward Operating Base, and the others were sourced in Houston. Samples of each brand were heated at $100^{\circ} \mathrm{C}$ for $1 \mathrm{~h}$. After the heat cycle, dimensions were measured while the samples squares were flat. Tests for time and temperature dependence on plastic sample dimensions were also performed on water bottle brands Baraka (average thickness $0.25 \mathrm{~mm}$ ) and Ozarka (average thickness $0.2 \mathrm{~mm}$ ):

1. Time dependence: heating at $100{ }^{\circ} \mathrm{C}$ for varying lengths of time.

2. Temperature dependence: heating for $5 \mathrm{~min}$ at temperatures ranging from 60 to $100{ }^{\circ} \mathrm{C}$.

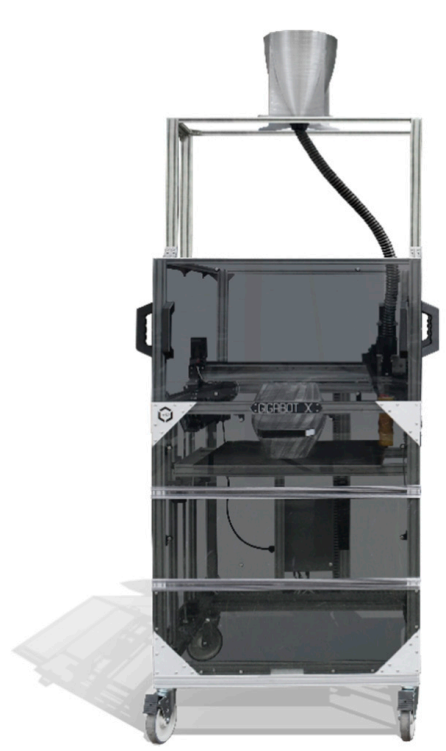

(a)

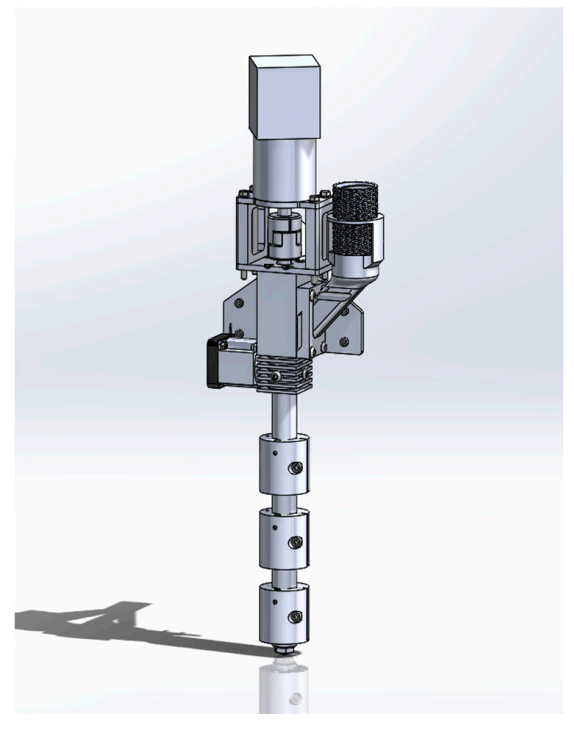

(b)

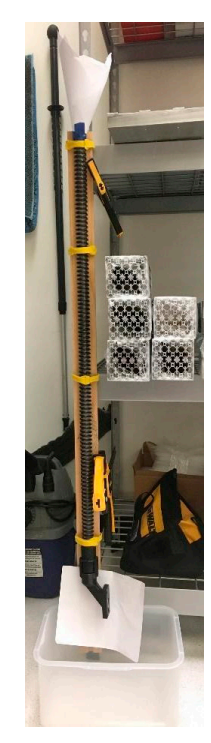

(c)

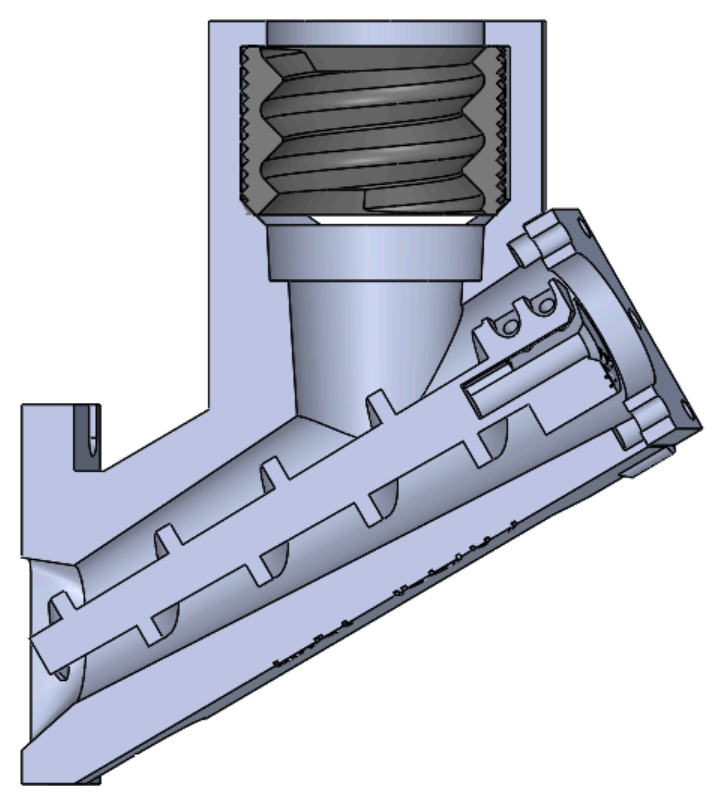

(d)

Figure 2. (a) Gigabot $X$ design with feed tube, (b) close up of 3-heat zone extruder, (c) feed test apparatus and (d) cross-section view of the Crammer feed throat with the auger screw inside. 
Finally, to improve feeding of water bottle rPET flake and other nonuniform regrind into the Gigabot X extruder, a Crammer (Figure 2d) was developed. The re:3D Crammer is a motorized auger screw that mounts onto the extruder and physically pushes the rPET flake from a feed tube and into the extruder. The Crammer's motor is synced with the pellet extruder motor via the duplicate nozzle, or ditto printing, feature of the open source Marlin firmware. This allows the Crammer to convey flake whenever the main extruder extrudes, and to scale the rate of material conveyance with the extrusion rate of the main extruder. The Crammer's components are all 3D printed from polycarbonate and were made available as open source designs [71].

Typical auger screws convey material with a screw that fits snugly in a barrel, preventing any material from passing between the screw flighting. This style of screw was tested with TPU pellets and successfully conveyed them. However, water bottle flake would get caught between the screw and the internal walls of the feed throat, stalling the Crammer motor and preventing conveyance. Since higher tolerance between the parts could not be achieved with 3D printing, an alternative screw was designed that left space between the screw threads and the feed throat internal walls, allowing flake to pass between the screw flighting without getting stuck (Figure $2 \mathrm{~d}$ ). This design was able to convey the water bottle flake without the flake getting trapped between any of the components.

A second feeding system approach was also tested on the granulated water bottles. A Gigabot X was outfitted with an extruder-mounted hopper, a $1.75 \mathrm{~mm}$ printer nozzle diameter, and a 3D-printed part cooling arrangement shown in Figure 3. The design files for the cooling setup can be found on the Open Science Framework [71].

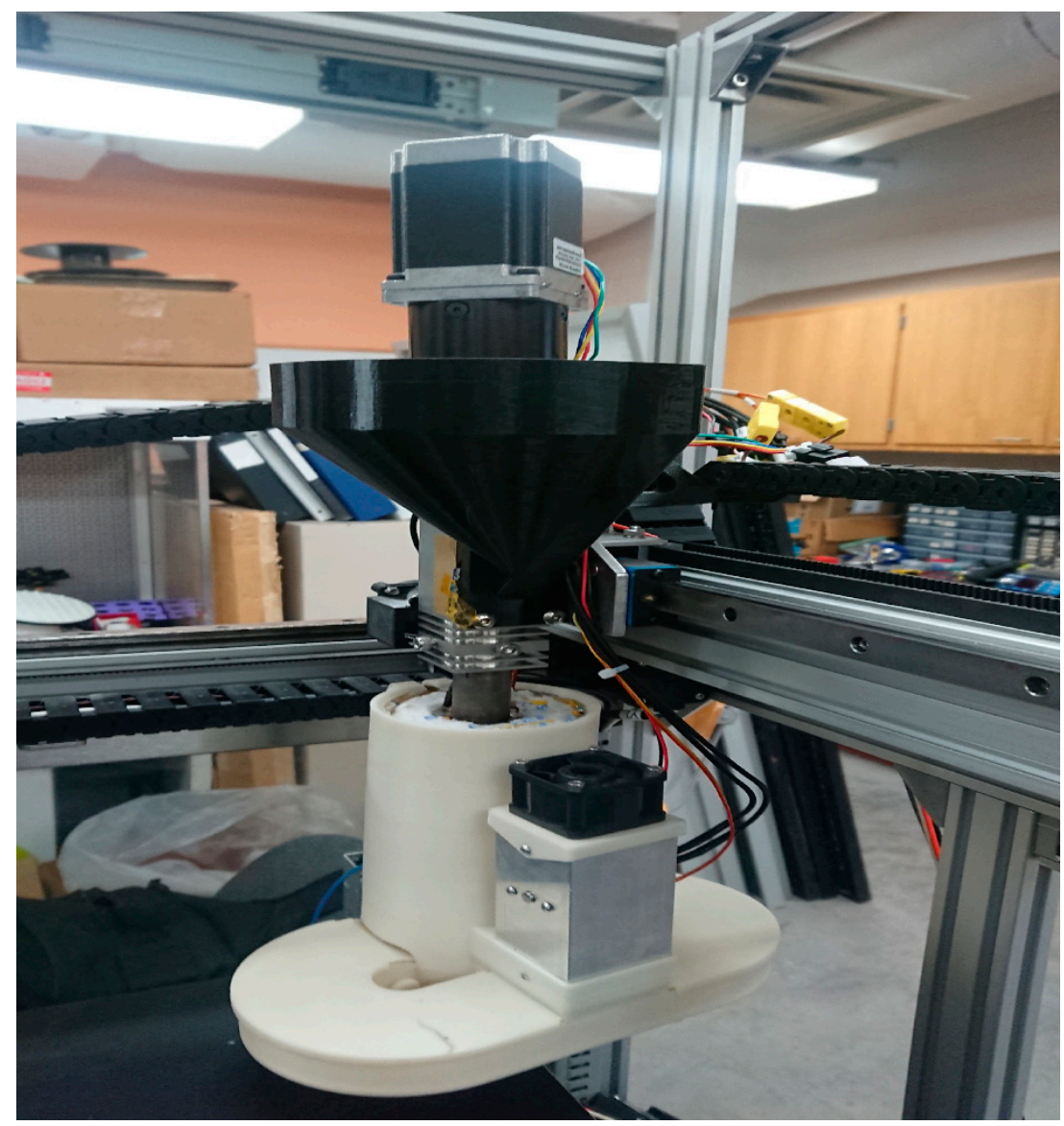

Figure 3. Gigabot $\mathrm{X}$ with 3D-printed direct feed hopper (black) and 3D-printed cooling shroud (white) with the source code available [71]. 


\subsection{Printing Settings Optimization}

Using the extruder-mounted hopper and 3D-printed cooling setup in Figure 3, 3D print optimization was performed on two geometries:

1. Cylinder: $20 \mathrm{~mm}$ diameter, $40 \mathrm{~mm}$ length, slicer generated mass of $17.3 \mathrm{~g}$.

2. Cuboid: $50 \mathrm{~mm}$ length, $50 \mathrm{~mm}$ width, $5 \mathrm{~mm}$ height, slicer generated mass of $17.3 \mathrm{~g}$.

Following similar protocols to those established by Woern et al. [61], optimization was performed in the 180 to $260^{\circ} \mathrm{C}$ region, with the heater region closer to the nozzle always having an equal or higher temperature. The minimum temperature at the top of the feeder was chosen based on the motor skipping. This indicates that the torque required to turn the extruder screw is higher than the extruder motor's torque output, which usually means material is unmelted or highly viscous. A temperature where there was no motor skipping was chosen $\left(210{ }^{\circ} \mathrm{C}\right)$. The maximum temperature close to the nozzle was chosen based on the blob-like appearance of the 3D print due to melting of the material $\left(240^{\circ} \mathrm{C}\right)$. Specimens were 3D printed at various temperature combinations in the selected "printable" region, and the chosen geometries were optimized based on visual quality and mass of the specimen. Three print speeds were tested: 10,30 and $50 \mathrm{~mm} / \mathrm{s}$.

\subsection{Mechanical Testing}

Two sets of mechanical testing took place. First, on the feed system shown in Figure 2 with rPET water bottle flake. Before loading into the $3 \mathrm{D}$ printer, the water bottle flake was dehydrated at $100^{\circ} \mathrm{F}$ for $24 \mathrm{~h}$, then placed in a $180^{\circ} \mathrm{C}$ oven for $5 \mathrm{~min}$ to improve feeding based on the results of the water bottle flake testing. With the Crammer, the water bottle flake could be extruded enough to produce ASTM D638 Type I tensile bars with a $0.8 \mathrm{~mm}$ nozzle and a $0.6033 \mathrm{~mm}$ layer height. Tensile bars were pulled on an Admet eXpert 2600 with a tension test with extensometer setup using the ASTM D638 testing standard. Five (5) specimens were tested in each sample. Specimens were massed on a digital scale.

Second, tensile testing was completed on the Ultrafuse rPET pellets (not PET flakes) using the ASTM D638 Type 1 standard tensile bars on the second set up in Figure 3. The nozzle size was $1.75 \mathrm{~mm}$. layer height $1 \mathrm{~mm}$. The bars were 3D printed at ideal print settings that were found during optimization (see Appendix A) of the cuboid at 100\% infill. The infill grid pattern was set to 45 degrees with respect to the long axis of the tensile bars. Five (5) specimens were tested in each sample. The specimens were then pulled until failure using a $10 \mathrm{kN}$ load cell on an Instron 4210 Testing machine and the speed of testing was $5 \mathrm{~mm} / \mathrm{min}$. The strain data were captured using the crosshead of the Instron 4210 . All mechanical testing was at room temperature $\left(23^{\circ} \mathrm{C}\right)$.

\section{Results and Discussion}

\subsection{Particle Size Analysis of Granulate and Feeding}

For the Ultrafuse rPET pellets shown in Figure 4, the average area in Figure 4a was $8.73 \mathrm{~mm}^{2}$ and the median area was $8.57 \mathrm{~mm}^{2}$, with a standard deviation of 4.59 . The average circularity for the Ultrafuse rPET in Figure $4 \mathrm{~b}$ was 0.47 and the median was 0.50, with a standard deviation of 0.25 .

As shown in Figure 5, the average area in Figure 5 a was $12.56 \mathrm{~mm}^{2}$ and the median area was $9.27 \mathrm{~mm}^{2}$, with a standard deviation of 10.43 . The average circularity for the unscreened water bottle rPET in Figure $5 \mathrm{~b}$ was 0.47 , the median was 0.49 , with a standard deviation of 0.17 .

By comparing the results of the two materials in Figures 4 and 5, the particle area of the Ultrafuse pellets is substantially smaller than the recycled water bottle granulate, as is the standard deviation. The circularity of the two materials is equivalent. A clear approach to improving the 3D printability of the recycled water bottle PET is simply to reduce its size. The impact of the four approaches to reduce the size of the rPET water bottle granulate is shown in Figure 6. 


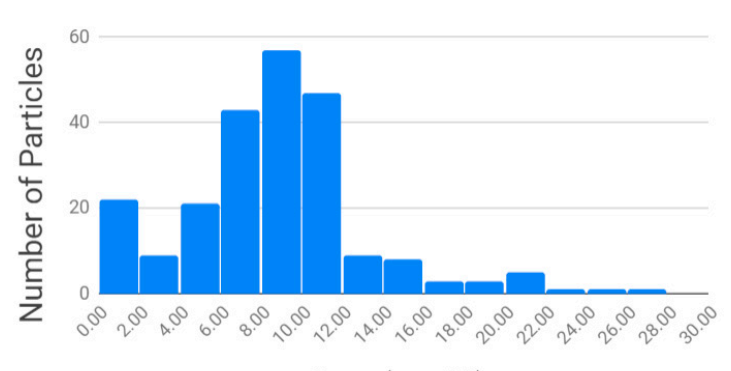

Area $\left(\mathrm{mm}^{\wedge} 2\right)$

(a)

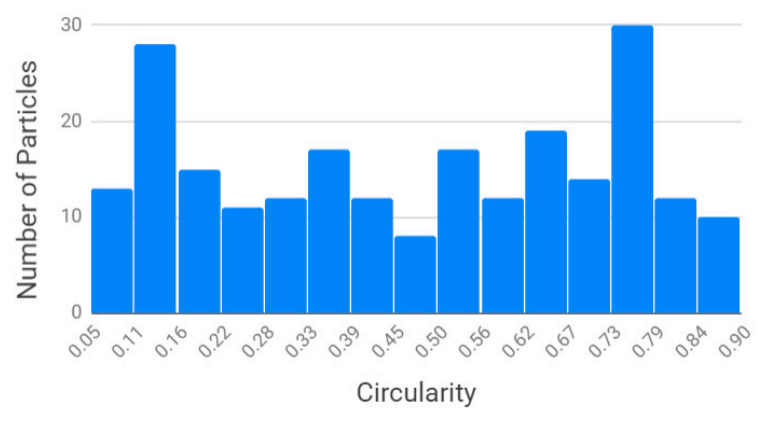

(b)

Figure 4. Ultrafuse rPET: particle size distribution (a) and particle circularity as a function of area (b).

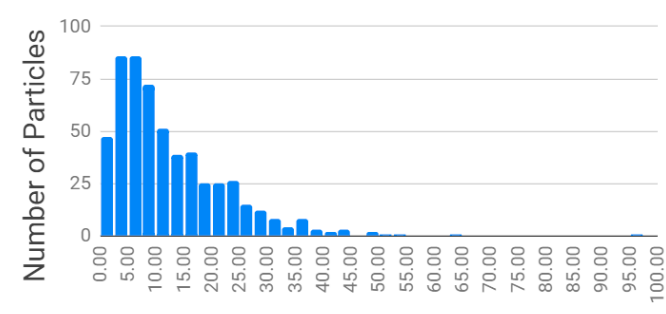

Area $\left(\mathrm{mm}^{\wedge} 2\right)$

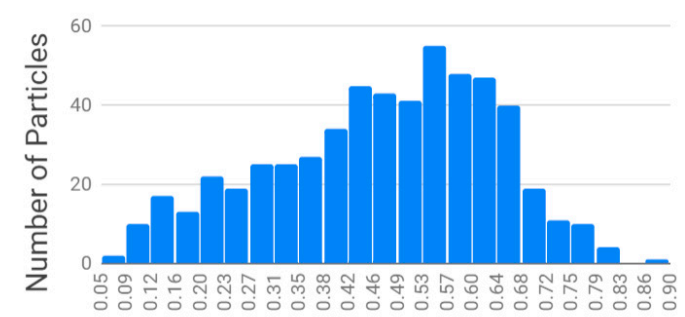

Circularity

(a)

(b)

Figure 5. Houston-sourced PET water bottle flake: particle size distribution (a) and particle circularity (b).

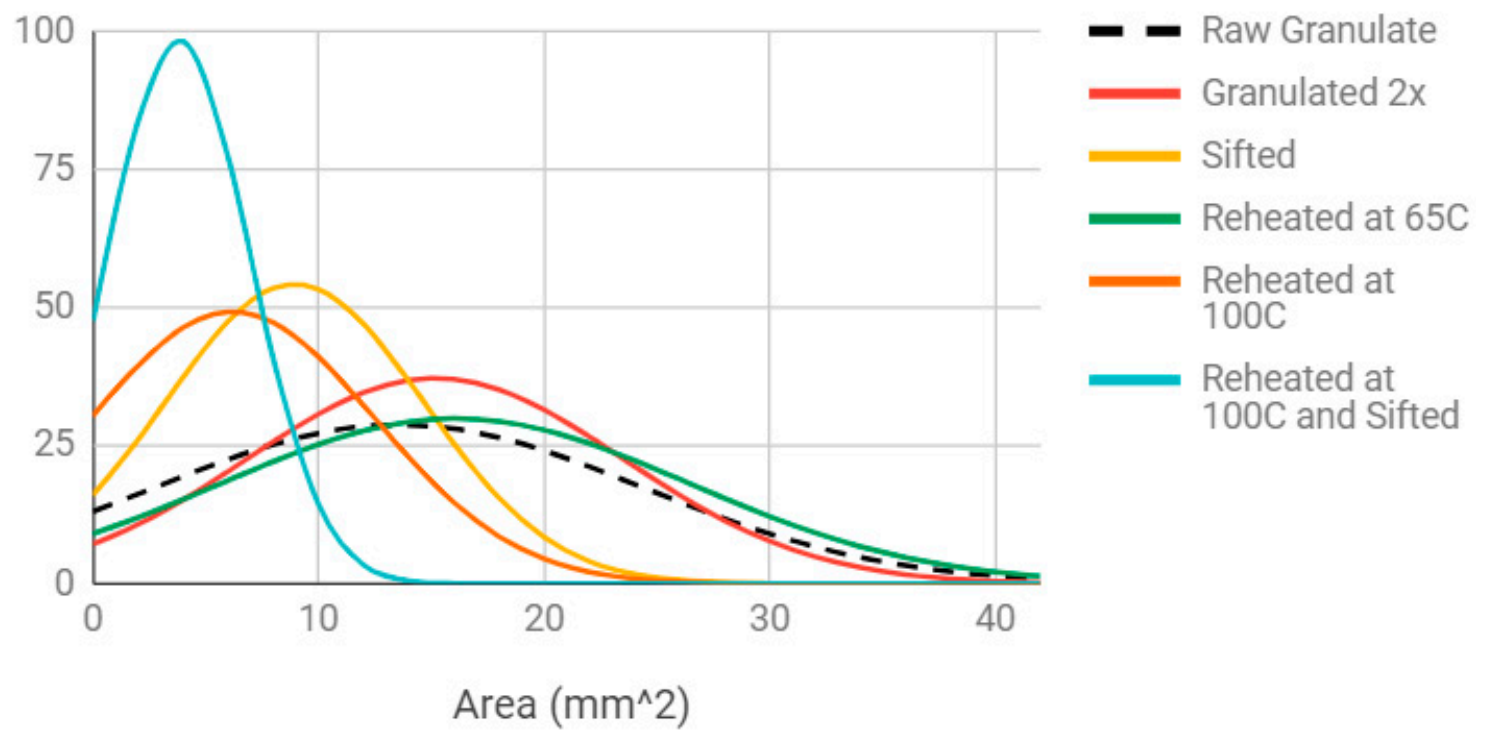

Figure 6. Effect of different processing methods on the normal distribution curves for PET water bottle granulate particle area.

As shown in Figure 6, the ImageJ particle analysis revealed the following conclusions for the different processing methods:

1. Granulating twice: Passing the water bottle granulate through the SHINI granulator twice does not decrease particle size (Figure 6). In fact, it shifts the particle size distribution to the right, toward larger particles. This indicates a loss of smaller particles $\left(<2 \mathrm{~mm}^{2}\right.$ in area) in the granulator. Not only does this processing step take more time and energy, it is ineffective. 
2. Sifting: Sifting successfully reduces the average particle area from 12.56 to $9.14 \mathrm{~mm}^{2}$ and shifts the particle size distribution curve to the left (Figure 6). This is a promising method for obtaining a 3D-printable granulate from rPET water bottles, but results in additional waste plastic.

3. Heating: Heating at $65^{\circ} \mathrm{C}$ does not reduce particle area and instead slightly shifts the normal distribution curve to the right (Figure 6). This may indicate a loss of small particles in the dehydrator during the heating process, since the smallest particles can fall through the dehydrator's screen holes. However, heating at $100{ }^{\circ} \mathrm{C}$ in the oven does reduce particle area (Figure 6), presumably because the flat plastic particles curl and contract in area while also increasing in thickness. The sample heated at $100^{\circ} \mathrm{C}$ also contained some particles that underwent a color change from clear to opaque white. The shape and color changes indicate crystallization of the amorphous PET water bottle plastic. Crystallization begins at the glass transition temperature $(\mathrm{Tg})$, which for PET is in the range of $153-178^{\circ} \mathrm{F}\left(67-81^{\circ} \mathrm{C}\right)$ [72]. This explains why the shape and color changes were present in the PET heated at $100^{\circ} \mathrm{C}$ (above Tg) and not in the PET heated at $65{ }^{\circ} \mathrm{C}$ (below Tg).

4. Combined sifting and $100{ }^{\circ} \mathrm{C}$ heating: Finally, the combined approach was shown to further tighten the particle size distribution and shift it towards smaller particles as shown in Figure 6.

To investigate the impact of different water bottle sources on rPET properties when heated, squares cut from various brands of water bottles were heated at $100{ }^{\circ} \mathrm{C}$ for $1 \mathrm{~h}$ (Figure 7). After heating, their dimensions were measured while the squares were flat, and the percent change was found (Table 1).

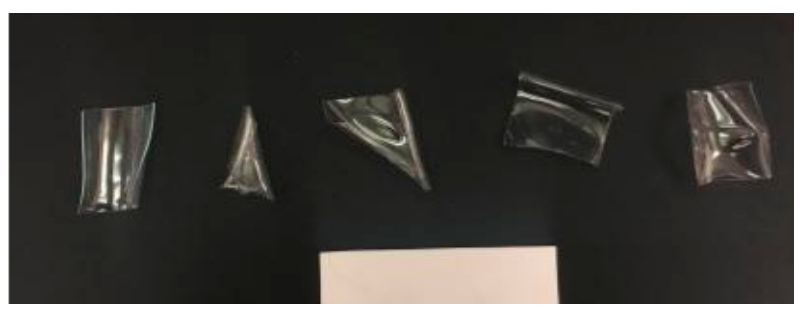

Figure 7. Water bottle squares after $1 \mathrm{~h}$ at $100{ }^{\circ} \mathrm{C}$ (scale shown with $50.8 \mathrm{~mm}$ white bar).

Table 1. The $25.4 \mathrm{~mm}$ water bottle squares before and after a $1 \mathrm{~h}$ heat cycle at $100{ }^{\circ} \mathrm{C}$.

\begin{tabular}{cccccc}
\hline & \multicolumn{2}{c}{ Post-Thermal Treatment } & \multicolumn{2}{c}{ Percent Change } \\
\hline Bottle Brand & Width $(\mathbf{m m})$ & Length $(\mathbf{m m})$ & Area $\left(\mathbf{m m}^{2}\right)$ & Width & Length \\
\hline Baraka & 17.8 & 23.9 & 424.5 & $-30.0 \%$ & $-6.0 \%$ \\
\hline Hill Country Fare & 22.1 & 23.1 & 510.8 & $-13.0 \%$ & $-9.0 \%$ \\
\hline Great Value & 21.3 & 24.4 & 520.3 & $-16.0 \%$ & $-4.0 \%$ \\
\hline Ozarka & 21.3 & 24.6 & 525.7 & $-16.0 \%$ & $-3.0 \%$ \\
\hline Texas Music Water & 22.4 & 24.6 & 550.7 & $-12.0 \%$ & $-3.0 \%$ \\
\hline
\end{tabular}

PET samples heated to $100{ }^{\circ} \mathrm{C}$ underwent significant contractions in length and width across all water bottle brands. The sample squares contracted different amounts in each dimension, with an average percent change of $-17.4 \%$ in one dimension and $-5 \%$ in the other (Table 1 ). The difference between the two dimensions may be caused by the water bottle manufacturing process, but more investigation is needed to confirm. This indicates that heating above the glass transition temperature shows promising results in improving particle shape.

Heating tests for time dependency on PET sample dimension show that area reduction occurs within the first five minutes, and additional heating time does not provide additional particle shape benefits (Figure 8a). By contrast, in Figure 8b, area reduces as temperature increases, beginning at the glass transition temperature (Tg) of PET. Area changes were similar across water bottle brands Baraka 
(avg thickness $0.25 \mathrm{~mm}$ ) and Ozarka (average thickness $0.2 \mathrm{~mm}$ ). These experiments also confirmed that the percent change in width was consistently double than that in length.

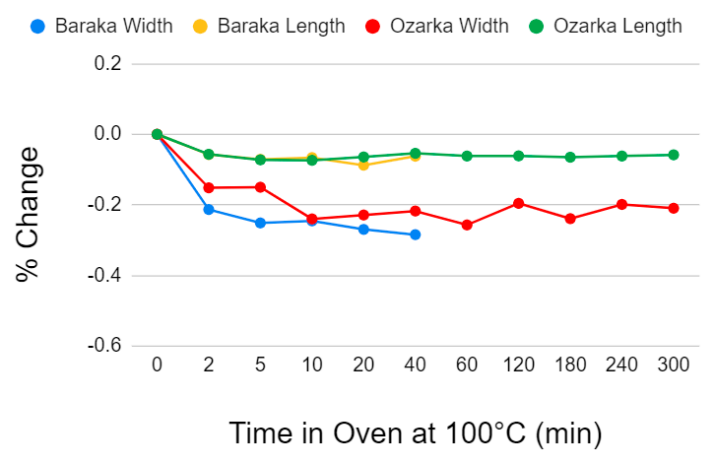

(a)

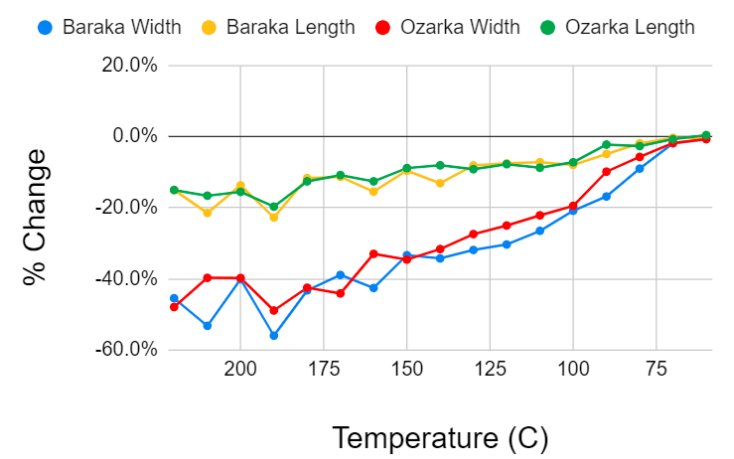

(b)

Figure 8. Effect of heating time on dimensions (width and length) of $25.4 \mathrm{~mm}$ squares of PET water bottle plastic heated at $100{ }^{\circ} \mathrm{C}$ (a) and the effect of temperature on dimensions (width and length) of $25.4 \mathrm{~mm}$ squares of PET water bottle plastic heated for $5 \mathrm{~min}(\mathbf{b})$.

Based on conclusions from the temperature and time dependence tests, a sample of rPET water bottle flake was sifted, then heated at $190^{\circ} \mathrm{C}$ for $5 \mathrm{~min}$ to obtain a sample with the smallest cross-sectional particle area (Figure 9 as compared to results in Figure 6).

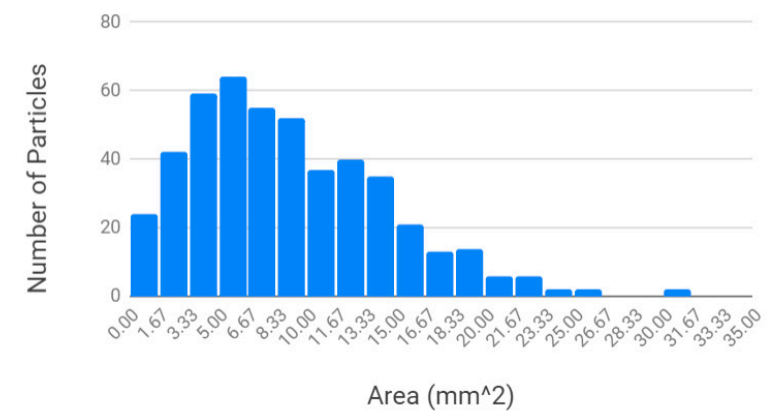

Figure 9. Particle size distribution of rPET water bottle flake sifted, then heated at $190{ }^{\circ} \mathrm{C}$ for $5 \mathrm{~min}$.

Although the combined sifting and heating at $190^{\circ} \mathrm{C}$ had the best chance of providing a functional material for the Gigabot X, the feeding tests showed that it was still incompatible. Although the Ultrafuse rPET pellets were easily fed through feed throat and $25.4 \mathrm{~mm}$ tubing, the processed samples of PET water bottle flake did not consistently feed through the system. This severely impacted 3D printability via the feed tube, and led to the development of the Crammer, or motorized auger screw (Figure 2), to physically push the water bottle flake from the feed tube and into the extruder. This system enabled post-consumer water bottle-sourced rPET flake to be directly 3D printed.

\subsection{Thermal Analysis}

Figure 10 show the DSC curve for a sample of PET water bottle $\mathrm{mW} / \mathrm{mg}$ as a function of temperature $\left({ }^{\circ} \mathrm{C}\right)$. The positive y axis indicates exothermic reactions, while downward designates endothermic reactions. The PET water bottle samples have endothermic peaks after approximately $250{ }^{\circ} \mathrm{C}$, which indicates a melting peak, showing that the PET flakes have a melting temperature of approximately $250^{\circ} \mathrm{C}$, which is what is expected of PET resin [73]. 


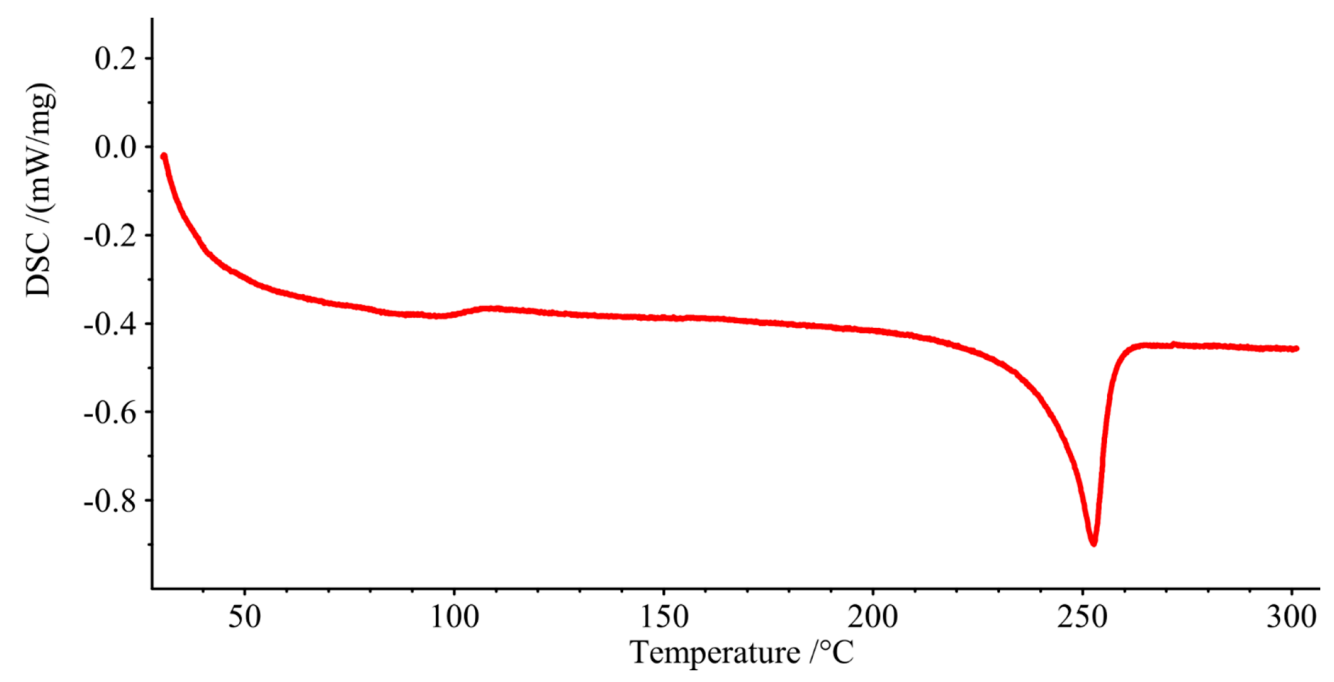

Figure 10. DSC curve of shredded water bottle.

\subsection{D Printing}

\subsubsection{Optimization Results}

The optimum temperature settings for 3D printing were found for the approach shown in Figure 3 in Table 2 (details of all runs available in Appendix A). It should be noted, however, that any specimen $3 \mathrm{D}$ printed in the given temperature range was sufficiently good in visual quality and mass of the 3D print.

Table 2. Optimal 3D print settings for the three temperature zones of the Gigabot $\mathrm{X}$ for no-fan and small-fan cases for rPET flake (Figure 3).

\begin{tabular}{ccccc}
\hline Cooling Fan & Shape of Print & \multicolumn{3}{c}{ Temperature $\left({ }^{\circ} \mathbf{C}\right)$} \\
\hline \multirow{2}{*}{ No fan used } & Cylinder & 210 & 200 & 200 \\
\hline \multirow{2}{*}{ Small fan used } & Cuboid & 230 & 230 & 220 \\
\hline & Cylinder & 220 & 220 & 210 \\
\hline
\end{tabular}

Specimens 3D printed at high speeds were consistently underextruded for various temperatures. Hence, a low speed of $10 \mathrm{~mm} / \mathrm{s}$ was chosen as the ideal print speed.

\subsubsection{Mechanical Testing}

Water bottle rPET flake was successfully 3D printed into tensile specimens (Figure 11) with an adapted Gigabot $X$ and Crammer shown in Figure 2. The average Ultimate Tensile Strength (UTS) of these direct 3D-printed materials was found to be $20.35 \mathrm{MPa}$, with a standard deviation of $0.187 \mathrm{MPa}$.

The variance in the UTS values and the resulting standard deviation may be a result of macro voids in the rPET tensile bars. These voids are caused by inconsistency in extrusion throughout a single 3D print (Figure 12). This caused a range in mass of the samples. The samples had an average mass of $10.4 \mathrm{~g}$, with a standard deviation of $0.76 \mathrm{~g}$. Optimization of the Crammer and further research into improving rPET flake extrusion may resolve the macro voids and improve the UTS of rPET flake to be more comparable with virgin PET, or rPET fabricated first into filament. 


\begin{tabular}{|cc|}
\hline 18 & 18 \\
\hline 19.1 & 19.1 \\
\hline 19.2 & 19.2 \\
\hline 19.4 & 19.4 \\
\hline 19.5 & 19.5 \\
\hline 19.6 & 19.6 \\
\hline 19.7 & 19.7 \\
\hline 19.8 & 19.8 \\
\hline 19.9 & 19.9 \\
\hline 20.1 & 20.1 \\
\hline
\end{tabular}

Figure 11. Tensile bars 3D printed with rPET water bottle flake on the adapted Gigabot $X$ and Crammer shown in Figure 2.

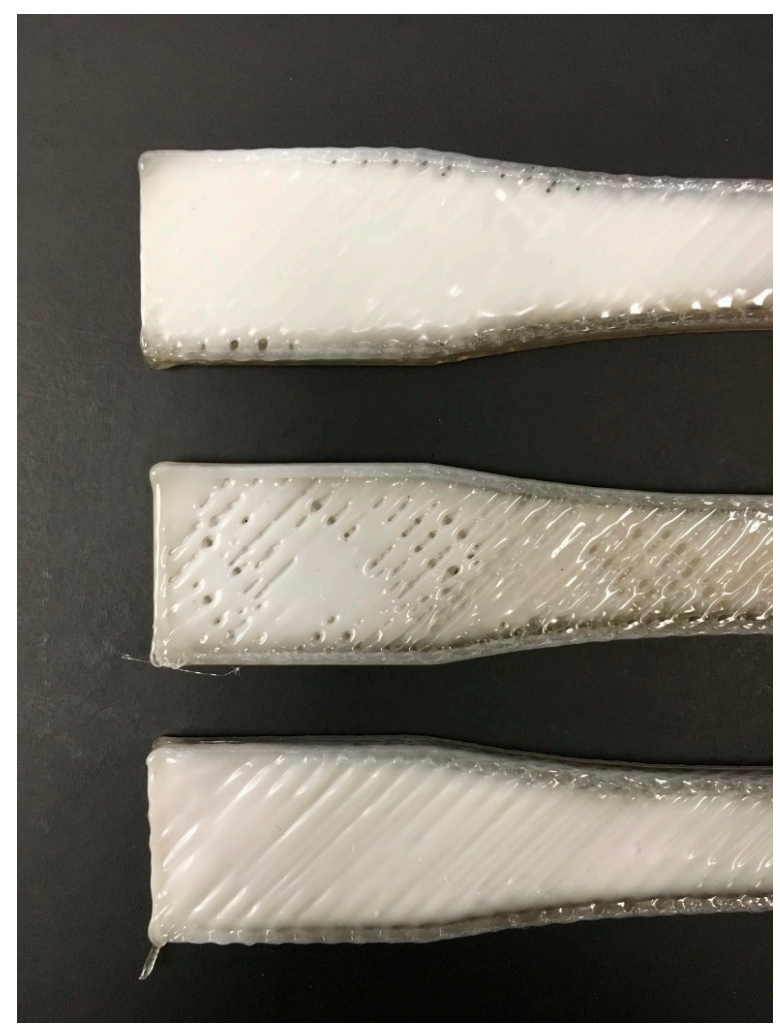

Figure 12. Close up of rPET water bottle tensile bars 3D printed with the same print settings. Inconsistent extrusion caused both under extrusion (top, middle) and over extrusion (bottom), with under extruded sections resulting in macro voids that decreased the UTS of the tensile bar.

In addition, using the second feed system (shown in Figure 3) was attempted. Although rPET from flake was found to be 3D printable via direct hopper, the lack of reproducibility and extreme brittleness resulted in a nonviable 3D print for tensile testing, since it could not be removed intact from the build surface, as shown in Figure 13. 


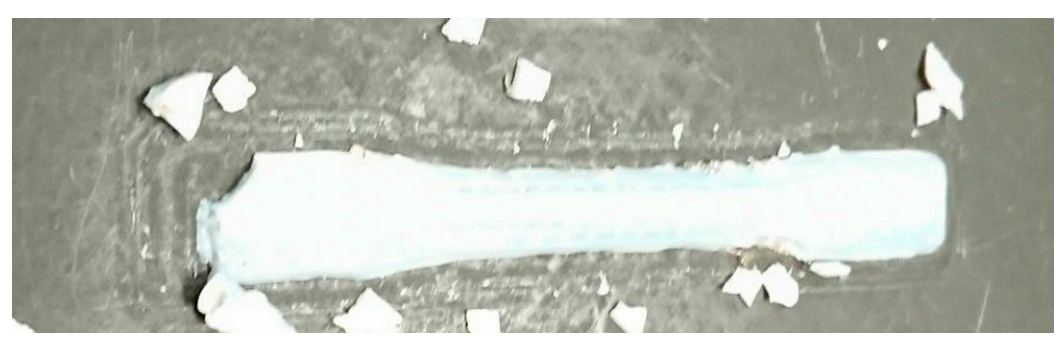

Figure 13. Tensile bar 3D printed in rPET from water bottle flake fractured on bed removal if 3D printed in humid open air.

Using the second feed system approach, Ultrafuse rPET pellets were tested with and without a cooling fan shown in Figure 3. The average tensile strength of the rPET pellets 3D printed with a cooling fan was $12.93 \mathrm{MPa}$, with a standard deviation of $4.72 \mathrm{MPa}$, while the average tensile strength of the sample 3D printed without using a cooling fan was $25.32 \mathrm{MPa}$, with a standard deviation of $5.82 \mathrm{MPa}$. The use of a cooling fan clearly reduced the tensile strength of the specimen although it provided more accurate $3 \mathrm{D}$ printing of small features as the forced cooling locked the molten plastic into the $3 \mathrm{D}$ form and reduced oozing. It is also observed that the average mass of the sample 3D printed using a cooling fan was $9.5 \mathrm{~g}$, while it was $9.2 \mathrm{~g}$ without using a fan. This mass discrepancy is most likely attributed to the variance in feeding and thus porosity of the direct feed process. The higher porosity in the cooling case reduced the UTS similar to the observed effect from inconsistent feeding observed for the first feed system approach and rPET flake.

To provide a comparison for the tensile bars 3D printed from water bottle flake, the Ultrafuse rPET pellets were also 3D printed on the first feed system setup (Figure 2) and the results were found to be more uniform. This resulted in a UTS of $29.62 \mathrm{MPa}$ and standard deviation of $4.43 \mathrm{MPa}$. The same nozzle $(0.8 \mathrm{~mm})$ layer height and layer settings were used for the rPET flake. The highest value observed is within the range previously reported for PET water bottles in a scientifically controlled environment using preformulated filament that enables better control of the material extrusion [60]. PET has a bulk tensile strength of 47 to $90 \mathrm{MPa}$ [74], whereas Zander et al. have shown that the strength of 3D-printed PET ranged from 27 to $45 \mathrm{MPa}$ [60]. Woern et al. have shown rPET in a Gigabot $\mathrm{X}$ prototype produced an average tensile strength was $40 \mathrm{MPa}$ [61]. Overall the UTS observed in this study for direct 3D printing water bottle flake was approximately equivalent to half that observed for Zander et al., which used a two-step process that first extruded filament and then 3D printed it. Zander et al. also showed that improved properties were possible forming waste composites [60], which is a clear potential to be successful for this direct shredded waste FPF 3D printing approach shown here. In addition, mixing rPET from flake and industrial rPET pellets or mixing with polypropylene to form blends [75] may lead to improved mechanical strength for FPF and can be investigated in the future.

Further study must be performed to understand the reason for this observed brittleness of the rPET from water bottle flakes as well as the variable strength of rPET pellets observed here although a few hypotheses can be made. First, the propensity of PET to break down in the presence of water and heat is well known. Although the PET was dried before entering the open hopper, the humidity in the room would have enabled access to water. Depending on the print order of the sample, the rPET plastic could have been held at elevated temperatures within the extruder of the Gigabot X, partially breaking it down. This may indicate why previous results with a shorter two stage Gigabot $X$ hot end (and thus a shorter high temperature residence time) resulted in higher tensile strengths for rPET [61]. This brings us to the second explanation - that the results indicate that there is wide variety in the quality of PET water bottle plastic and this plastic could have been of the less mechanically or chemically stable variety. The most perplexing result is that the strengths with cooling were approximately half those without cooling. This is not expected as a faster cooling rate would typically result in higher strength. The average mass with cooling was also larger by approximately $3 \%$, which would have also indicated 
that it would be stronger. The nature of material extrusion-based 3D printing may also help to explain this result. The observed macro porosity is a strong indicator that this was the primary explanation of both results. If the plastic in both cases was approximately the same (or even slightly higher for the cooling fan case), the rapid cooling could create more interline spacing (triangular shaped air gaps). As previously observed in FFF printing [76], these gaps would be expected to reduce strength even if the $3 \mathrm{D}$ prints appeared solid. In addition, because the two cases that were tested for tensile strength were no cooling and modest cooling, slow print speeds were necessary $(10 \mathrm{~mm} / \mathrm{s})$, which would be expected to increase any breakdown in the material in the 3D printer.

\subsubsection{Example Print}

The three-heating stage Gigabot $X$ was able to fabricate several example 3D prints with Ultrafuse rPET pellets. Despite the rPET being substantially weaker than injection molded PET, the values of 19.5 and $25 \mathrm{MPa}$ are close to those observed for commercial FDM of ABS plastic as well as FFF ABS 3D printed under realistic conditions [77]. This makes the rPET even FPF 3D printed directly from flake more than adequate for a number of applications. Several examples are shown for military tools and training aids in Figure 14: (A) Air Force training aid: successfully 3D printed with a 0.8 mm nozzle and no support; (B) KMZ topographical map: 3D printed first with a $1.75 \mathrm{~mm}$ nozzle, then a $0.8 \mathrm{~mm}$ nozzle to improve resolution; (C) propeller: 3D printed with a $0.8 \mathrm{~mm}$ nozzle with support (surfaces contacting the support can be improved with higher resolution and dual extrusion); (D) planning tool: the combination of support and high-detail parts could not be achieved with the resolution from either the 1.75 or $0.8 \mathrm{~mm}$ nozzles; (E) jet engine jig to paint the white line on a spinner: successfully 3D printed with a $1.75 \mathrm{~mm}$ nozzle with vase mode. Overhanging edges can be further improved by a smaller nozzle and this would also solve the quality issues shown in Figure 14B.

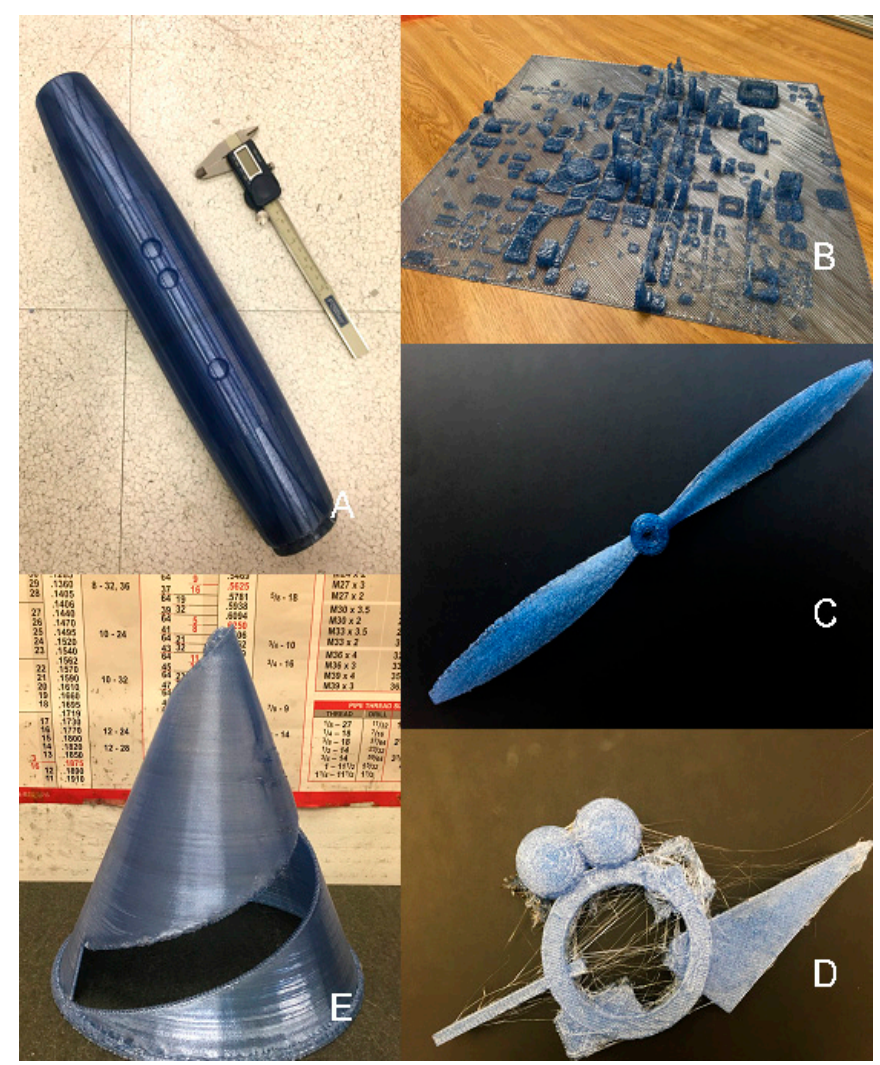

Figure 14. Example Gigabot $X$ test 3D prints: (A) Air Force training aid, (B) topographical map KMZ, (C) propeller, (D) planning tool, and (E) spinner. 
To further demonstrate the feasibility of using rPET to 3D print a high-demand object [78-81], the Gigabot $X$ was used to 3D print a face shield as shown in Figure 15.

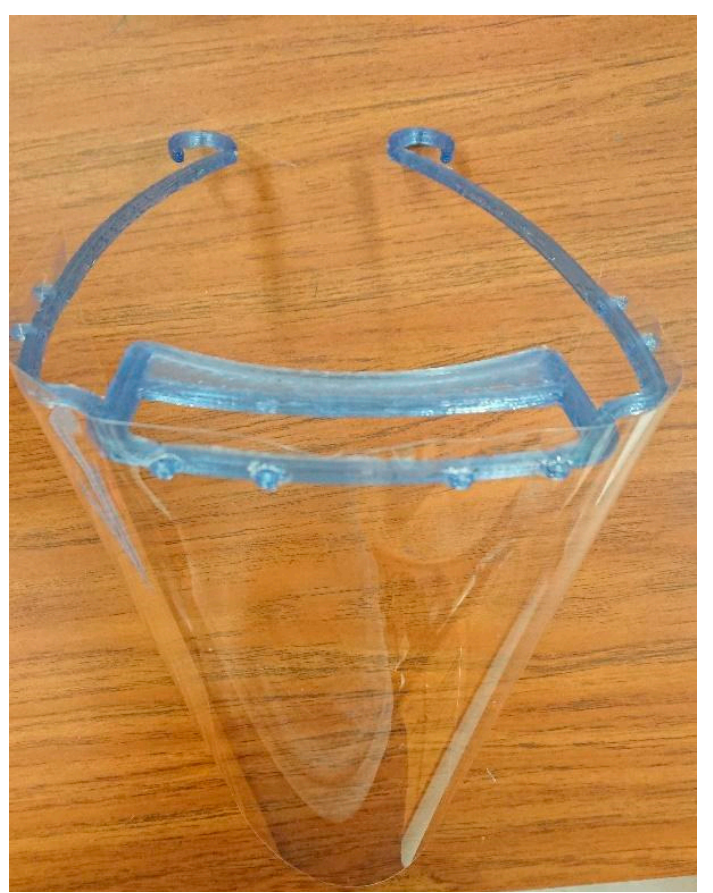

Figure 15. Recycled PET pellets used to 3D print a face shield.

\section{Future Work}

This study has uncovered several areas of future work. First, improved methods of granulating PET from water bottles as well as processing rPET flake into an FPF/FGF machine are needed. This is expected to help feeding issues observed in this study with the Gigabot $X$ feeding tube system which, unlike the extruder-mounted hopper system, enables large-scale, long-term 3D printing, and already works with uniform feedstocks such as pellets. Future investigation into improving feeding issues for water bottle flake and similarly shaped recycled flake can include flake processing techniques, feed system part geometry to improve flow, and improvements to the motorized auger screw to physically pack particles into the extruder. Second, the results of this study showed that there is a large difference in the rPET with different waste streams, processing history, and physical form (flakes or pellets). This could be a function not only of the supplier and their feedstocks and additives, but could also be influenced by age of the waste, whether it was stored in direct sunlight, and the thermal history. This represents a substantial challenge to the optimization of the DRAM process. One approach to partially solving this challenge is to expand the 'Consumer Bill of Rights' to include material ingredient lists maintained in a freely accessible digital database for all consumer products [82]. In addition, these rPET materials should be subjected to detailed rheological analysis and the necessity of viscosity enhancing additives can be explored. This can be for the pure rPET materials as well as mixtures of pellets and shredded water bottles. This is a complex problem and a far more detailed study should be completed looking at rPET from many sources, locations in the world, and suppliers to provide optimal 3D printing parameters for direct extrusion 3D printers such as the Gigabot X. One of the first steps could be the development of an open source melt flow index (MFI) device that could be used to rapidly screen rPET materials at a low cost. This would partially overcome the challenge of not knowing the history of a rPET material (even if the full chemical makeup was supplied by the manufacturers).

Going beyond more complete knowledge of the rPET material, the FPF 3D printing process can also be improved. Additional work is also needed to quantify the impact of nozzle height and layer 
height on the strength of rPET 3D prints as well. In order to overcome the slow printing speeds used in this study, a more powerful fan could be added to the system to enable rapid part cooling. This would be expected to allow for faster 3D printing (reducing residence time and reducing material breakdown). Detailed study measuring the crystallinity of the 3D-printed specimen is required to understand the reason for the difference in tensile strength between specimens 3D printed with and without a fan. To better understand the material breakdown, a careful study of residence time vs. strength could be completed for future work. In addition, as with PLA [29], ABS [83], and HDPE [84], the impact of the number of recycle loops should be investigated and compared to a filament-extruding approach for multiple cycles. This is important to have a closed-loop supply chain in the circular economy [12,85]. It is likely that the direct rPET FPF 3D printing demonstrated in this study would provide an advantage, as the number of melt/solidification loops would be reduced by $1 / 2$. Finally, in order to ensure that the rPET remains dry, a heated hopper/feeding unit could be investigated and would be expected to improve results. This work should enable rPET from water bottles to be used as a reliable feedstock for DRAM.

\section{Conclusions}

Although far from optimized, the results of this study show the potential to reach a circular economy for post-consumer recycled rPET as a DRAM feedstock when used with Gigabot X FPF/FGF 3D printing. The results showed that extended feeding tubes were challenging with rPET flakes when processed by simple shredding, sifting, or heating (and the combination), but they could be 3D printed using a Crammer to improve feeding, and resolution could be improved with active cooling. Further, this study showed a wide disparity in the physical properties of rPET depending on source and particle shape (flake or pellet) and indicated a large area for future work both in material characterization as well as processing and machine design to make rPET from water bottles a common feedstock.

Author Contributions: Conceptualization, S.L.S. and J.M.P.; data curation, N.G.T. and M.J.R.; formal analysis, H.A.L., N.G.T., M.J.R., M.J.F., S.L.S. and J.M.P.; funding acquisition, S.L.S. and J.M.P.; investigation, H.A.L., N.G.T. and M.J.R.; methodology, H.A.L., N.G.T. and M.J.R.; project administration, J.M.P.; resources, J.M.P.; supervision, J.M.P.; validation, H.A.L. and M.J.R.; visualization, H.A.L., N.G.T. and M.J.R.; writing-original draft, H.A.L. and J.M.P.; writing-review and editing, H.A.L., N.G.T., M.J.R., M.J.F., S.L.S. and J.M.P. All authors have read and agreed to the published version of the manuscript.

Funding: This research was funded by NSF SBIR Phase II, grant number: 1746480, and the WeWork Global Creator Award.

Acknowledgments: The authors would like to thank Aubrey Woern and Samantha Reeve for helpful comments.

Conflicts of Interest: Helen A. Little, Matthew J. Fiedler and Samantha L. Snabes are employees of re:3D, which manufactures the Gigabot X that was used in this study. The authors from MTU have no conflict of interest. The funders played no part in the design of the study, in the collection, analyses, or interpretation of the data, in the writing of the manuscript, or in the decision to publish the results.

\section{Appendix A}

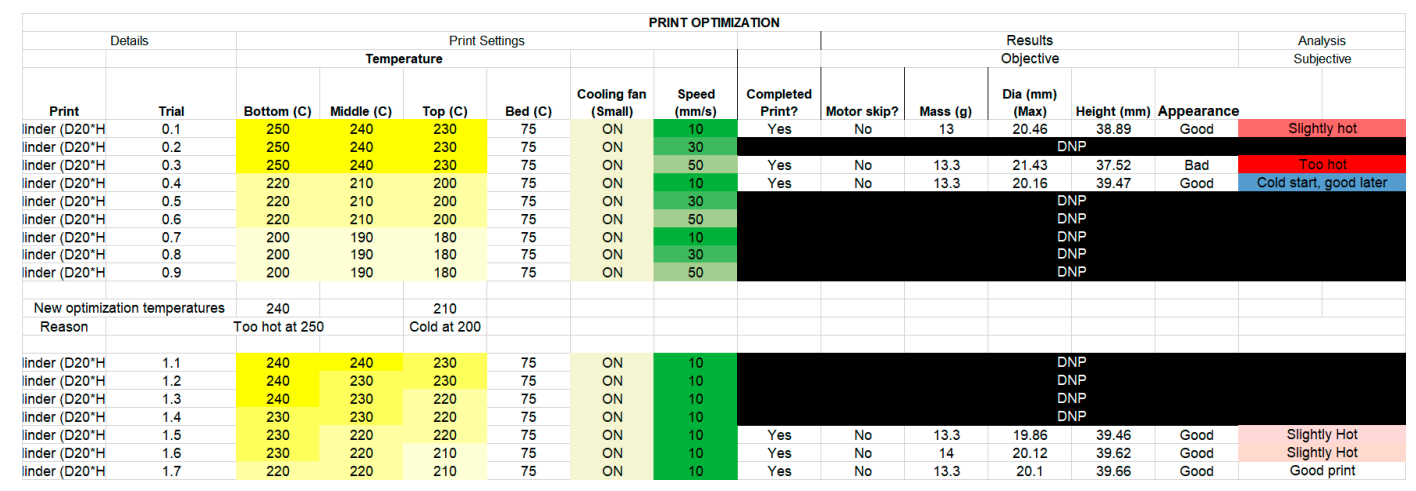

Figure A1. Print optimization with small-fan cylinder. 


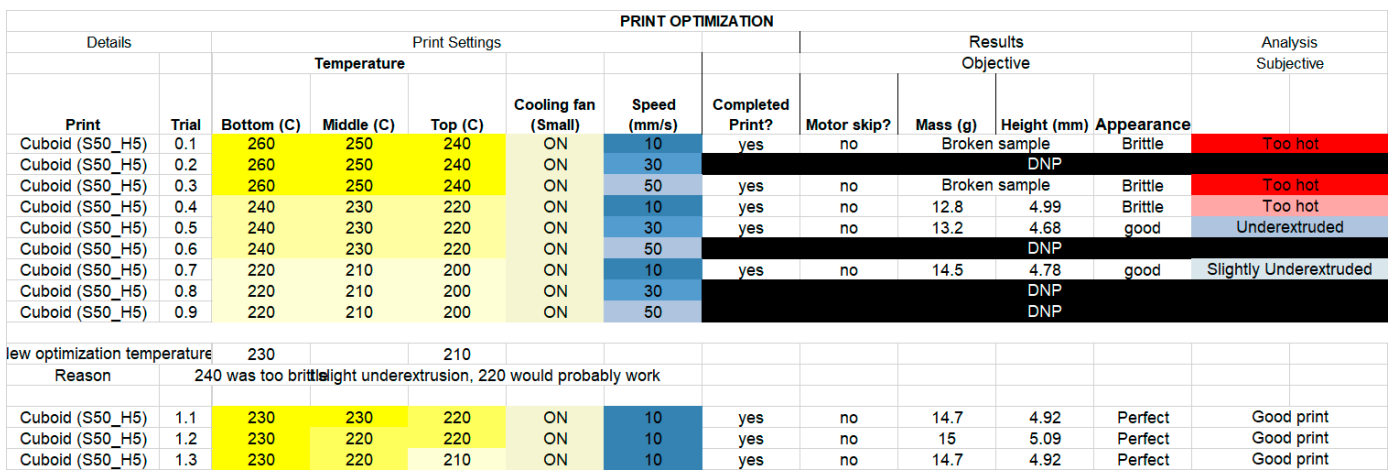

Figure A2. Print optimization with small-fan, large short cuboid.

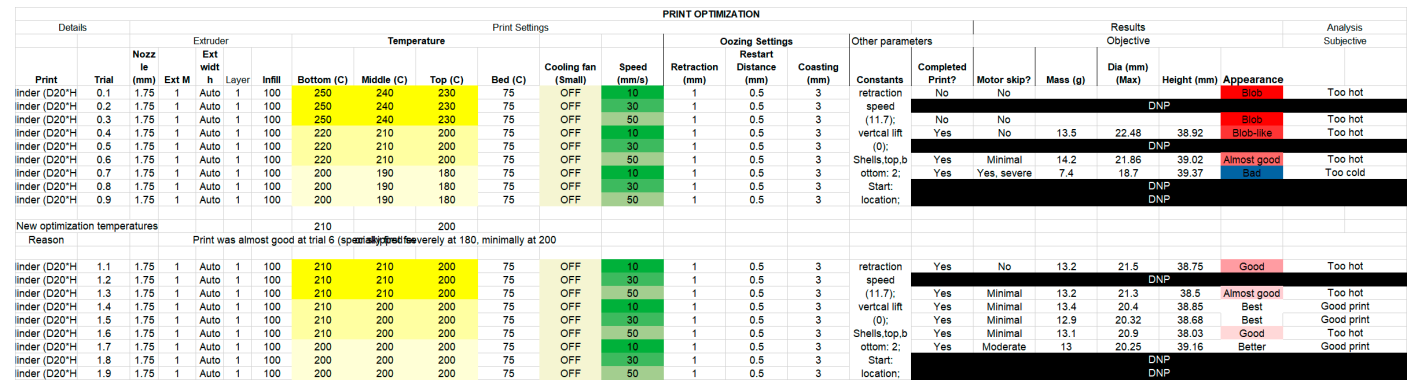

Figure A3. Print optimization with no-fan, small tall cylinder.

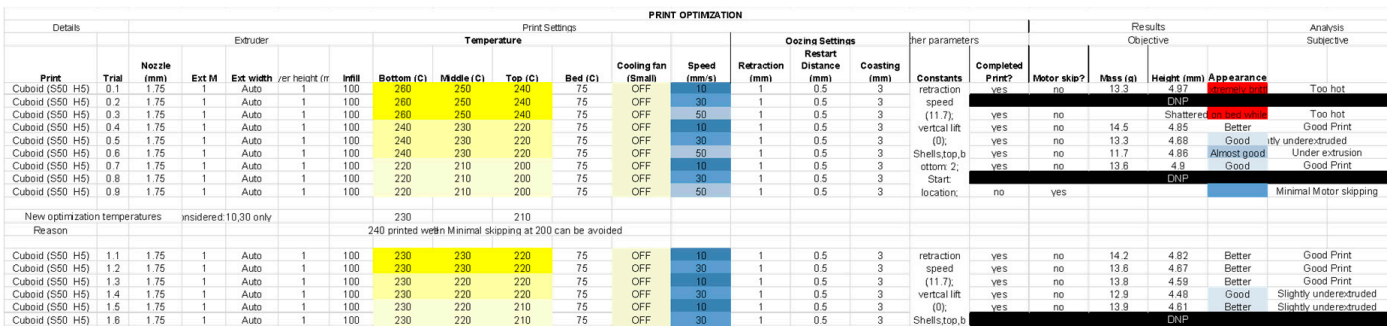

Figure A4. Print optimization with no-fan, large short cuboid.

\section{References}

1. Geyer, R.; Jambeck, J.R.; Law, K.L. Production, use, and fate of all plastics ever made. Sci. Adv. 2017, 3, e1700782. [CrossRef] [PubMed]

2. Brooks, A.L.; Wang, S.; Jambeck, J.R. The Chinese import ban and its impact on global plastic waste trade. Sci. Adv. 2018, 4, eaat0131. [CrossRef] [PubMed]

3. Katz, C. Piling Up: How China's Ban on Importing Waste Has Stalled Global Recycling. Available online: https://e360.yale.edu/features/piling-up-how-chinas-ban-on-importing-waste-has-stalledglobal-recycling (accessed on 8 May 2020).

4. McNaugton, S. How China's Plastic Waste Ban Forced a Global Recycling Reckoning. Available online: https://www.nationalgeographic.com/magazine/2019/06/china-plastic-waste-banimpacting-countries-worldwide/ (accessed on 8 May 2020).

5. Joyce, C.U.S. Recycling Industry Is Struggling to Figure Out A Future without China. Available online: https:/www.npr.org/2019/08/20/750864036/u-s-recycling-industry-is-struggling-to-figureout-a-future-without-china (accessed on 8 May 2020).

6. Corkery, M. As Costs Skyrocket, More U.S. Cities Stop Recycling. The New York Times, 17 March 2019; A.1.

7. Packaging Europe Pioneering Sorting Technology: HolyGrail Project Moves towards a Circular Economy. Available online: https:/packagingeurope.com/api/content/6c4a9c8e-81de-11e9-898c12f1225286c6/ (accessed on 8 May 2020).

8. Geissdoerfer, M.; Savaget, P.; Bocken, N.M.P.; Hultink, E.J. The Circular Economy-A new sustainability paradigm? J. Clean. Prod. 2017, 143, 757-768. [CrossRef] 
9. Kirchherr, J.; Reike, D.; Hekkert, M. Conceptualizing the circular economy: An analysis of 114 definitions. Resour. Conserv. Recycl. 2017, 127, 221-232. [CrossRef]

10. Stahel, W.R. The circular economy. Nat. News 2016, 531, 435. [CrossRef]

11. Zhong, S.; Pearce, J.M. Tightening the loop on the circular economy: Coupled distributed recycling and manufacturing with recyclebot and RepRap 3-D printing. Resour. Conserv. Recycl. 2018, 128, 48-58. [CrossRef]

12. Pavlo, S.; Fabio, C.; Hakim, B.; Mauricio, C. 3D-Printing Based Distributed Plastic Recycling: A Conceptual Model for Closed-Loop Supply Chain Design. In Proceedings of the 2018 IEEE International Conference on Engineering, Technology and Innovation (ICE/ITMC), Stuttgart, Germany, 17-20 June 2018; pp. 1-8.

13. Cruz Sanchez, F.A.; Boudaoud, H.; Camargo, M.; Pearce, J.M. Plastic recycling in additive manufacturing: A systematic literature review and opportunities for the circular economy. J. Clean. Prod. 2020, 264, 121602. [CrossRef]

14. Gwamuri, J.; Wittbrodt, B.T.; Anzalone, N.C.; Pearce, J.M. Reversing the Trend of Large Scale and Centralization in Manufacturing: The Case of Distributed Manufacturing of Customizable 3-D-Printable Self-Adjustable Glasses; Social Science Research Network: Rochester, NY, USA, 2014.

15. Wittbrodt, B.T.; Glover, A.G.; Laureto, J.; Anzalone, G.C.; Oppliger, D.; Irwin, J.L.; Pearce, J.M. Life-cycle conomic analysis of distributed manufacturing with open-source 3-D printers. Mechatronics 2013, 23, 713-726. [CrossRef]

16. Petersen, E.E.; Pearce, J. Emergence of Home Manufacturing in the Developed World: Return on Investment for Open-Source 3-D Printers. Technologies 2017, 5, 7. [CrossRef]

17. Petersen, E.E.; Kidd, R.W.; Pearce, J.M. Impact of DIY Home Manufacturing with 3D Printing on the Toy and Game Market. Technologies 2017, 5, 45. [CrossRef]

18. Laplume, A.O.; Petersen, B.; Pearce, J.M. Global value chains from a 3D printing perspective. J. Int. Bus. Stud. 2016, 47, 595-609. [CrossRef]

19. Baechler, C.; DeVuono, M.; Pearce, J.M. Distributed recycling of waste polymer into RepRap feedstock. Rapid Prototyp. J. 2013, 19, 118-125. [CrossRef]

20. Woern, A.L.; McCaslin, J.R.; Pringle, A.M.; Pearce, J.M. RepRapable Recyclebot: Open source 3-D printable extruder for converting plastic to 3-D printing filament. HardwareX 2018, 4, e00026. [CrossRef]

21. Kreiger, M.; Anzalone, G.C.; Mulder, M.L.; Glover, A.; Pearce, J.M. Distributed Recycling of Post-Consumer Plastic Waste in Rural Areas. MRS Online Proc. Libr. Arch. 2013, 1492, 91-96. [CrossRef]

22. Kreiger, M.A.; Mulder, M.L.; Glover, A.G.; Pearce, J.M. Life cycle analysis of distributed recycling of post-consumer high density polyethylene for 3-D printing filament. J. Clean. Prod. 2014, 70, 90-96. [CrossRef]

23. Zhong, S.; Rakhe, P.; Pearce, J.M. Energy Payback Time of a Solar Photovoltaic Powered Waste Plastic Recyclebot System. Recycling 2017, 2, 10. [CrossRef]

24. Sells, E.; Bailard, S.; Smith, Z.; Bowyer, A.; Olliver, V. RepRap: The Replicating Rapid Prototyper: Maximizing Customizability by Breeding the Means of Production. In Handbook of Research in Mass Customization and Personalization; World Scientific Publishing Company: Singapore, 2009; pp. 568-580. ISBN 978-981-4280-25-9.

25. Jones, R.; Haufe, P.; Sells, E.; Iravani, P.; Olliver, V.; Palmer, C.; Bowyer, A. RepRap-the replicating rapid prototyper. Robotica 2011, 29, 177-191. [CrossRef]

26. Bowyer, A. 3D Printing and Humanity's First Imperfect Replicator. 3D Print. Addit. Manuf. 2014, 1, 4-5. [CrossRef]

27. Hunt, E.J.; Zhang, C.; Anzalone, N.; Pearce, J.M. Polymer recycling codes for distributed manufacturing with 3-D printers. Resour. Conserv. Recycl. 2015, 97, 24-30. [CrossRef]

28. Sanchez, F.A.C.; Lanza, S.; Boudaoud, H.; Hoppe, S.; Camargo, M. Polymer Recycling and Additive Manufacturing in an Open Source context: Optimization of processes and methods. In Proceedings of Annual International Solid Freeform Fabrication Symposium; ISSF: Austin, TX, USA, 2015; p. 1591.

29. Cruz Sanchez, F.A.; Boudaoud, H.; Hoppe, S.; Camargo, M. Polymer recycling in an open-source additive manufacturing context: Mechanical issues. Addit. Manuf. 2017, 17, 87-105. [CrossRef]

30. Anderson, I. Mechanical Properties of Specimens 3D Printed with Virgin and Recycled Polylactic Acid. 3D Print. Addit. Manuf. 2017, 4, 110-115. [CrossRef]

31. Pakkanen, J.; Manfredi, D.; Minetola, P.; Iuliano, L. About the Use of Recycled or Biodegradable Filaments for Sustainability of 3D Printing. In Proceedings of the Sustainable Design and Manufacturing 2017; Campana, G., Howlett, R.J., Setchi, R., Cimatti, B., Eds.; Springer International Publishing: Cham, Switzerland, 2017; pp. 776-785. 
32. Mohammed, M.I.; Wilson, D.; Gomez-Kervin, E.; Tang, B.; Wang, J. Investigation of Closed-Loop Manufacturing with Acrylonitrile Butadiene Styrene over Multiple Generations Using Additive Manufacturing. ACS Sustain. Chem. Eng. 2019, 7, 13955-13969. [CrossRef]

33. Mohammed, M.I.; Wilson, D.; Gomez-Kervin, E.; Vidler, C.; Rosson, L.; Long, J. The Recycling of E-Waste ABS Plastics by Melt Extrusion and 3D Printing Using Solar Powered Devices as a Transformative Tool for Humanitarian Aid. In Proceedings of the 29th Annual International Solid Freeform Fabrication Symposium, Austin, TX, USA, 13-15 August 2018; pp. 80-92.

34. Mohammed, M.I.; Wilson, D.; Gomez-Kervin, E.; Rosson, L.; Long, J. EcoPrinting: Investigation of Solar Powered Plastic Recycling and Additive Manufacturing for Enhanced Waste Management and Sustainable Manufacturing. In Proceedings of the 2018 IEEE Conference on Technologies for Sustainability (SusTech), Long Beach, CA, USA, 11-13 November 2018; pp. 1-6.

35. Boldizar, A.; Möller, K. Degradation of ABS during repeated processing and accelerated ageing. Polym. Degrad. Stab. 2003, 81, 359-366. [CrossRef]

36. Chong, S.; Pan, G.-T.; Khalid, M.; Yang, T.C.-K.; Hung, S.-T.; Huang, C.-M. Physical Characterization and Pre-assessment of Recycled High-Density Polyethylene as 3D Printing Material. J. Polym. Environ. 2017, 25, 136-145. [CrossRef]

37. Pepi, M.; Zander, N.; Gillan, M. Towards Expeditionary Battlefield Manufacturing Using Recycled, Reclaimed, and Scrap Materials. JOM 2018, 70, 2359-2364. [CrossRef]

38. Woern, A.L.; Pearce, J.M. Distributed Manufacturing of Flexible Products: Technical Feasibility and Economic Viability. Technologies 2017, 5, 71. [CrossRef]

39. Hart, K.R.; Frketic, J.B.; Brown, J.R. Recycling meal-ready-to-eat (MRE) pouches into polymer filament for material extrusion additive manufacturing. Addit. Manuf. 2018, 21, 536-543. [CrossRef]

40. Reich, M.J.; Woern, A.L.; Tanikella, N.G.; Pearce, J.M. Mechanical Properties and Applications of Recycled Polycarbonate Particle Material Extrusion-Based Additive Manufacturing. Materials 2019, 12, 1642. [CrossRef]

41. Oblak, P.; Gonzalez-Gutierrez, J.; Zupančič, B.; Aulova, A.; Emri, I. Processability and mechanical properties of extensively recycled high density polyethylene. Polym. Degrad. Stab. 2015, 114, 133-145. [CrossRef]

42. Lee, J.H.; Lim, K.S.; Hahm, W.G.; Kim, S.H. Properties of recycled and virgin poly(ethylene terephthalate) blend fibers. J. Appl. Polym. Sci. 2013, 128, 1250-1256. [CrossRef]

43. Tian, X.; Liu, T.; Wang, Q.; Dilmurat, A.; Li, D.; Ziegmann, G. Recycling and remanufacturing of 3D printed continuous carbon fiber reinforced PLA composites. J. Clean. Prod. 2017, 142, 1609-1618. [CrossRef]

44. Parandoush, P.; Lin, D. A review on additive manufacturing of polymer-fiber composites. Compos. Struct. 2017. [CrossRef]

45. Heller, B.P.; Smith, D.E.; Jack, D.A. Planar deposition flow modeling of fiber filled composites in large area additive manufacturing. Addit. Manuf. 2019, 25, 227-238. [CrossRef]

46. Pringle, A.M.; Rudnicki, M.; Pearce, J.M. Wood Furniture Waste-Based Recycled 3-D Printing Filament. For. Prod. J. 2017, 68, 86-95. [CrossRef]

47. Zander, N.E. Recycled Polymer Feedstocks for Material Extrusion Additive Manufacturing. In Polymer-Based Additive Manufacturing: Recent Developments; ACS Symposium Series; American Chemical Society: Washington, DC, USA, 2019; Volume 1315, pp. 37-51. ISBN 978-0-8412-3426-0.

48. Dertinger, S.C.; Gallup, N.; Tanikella, N.G.; Grasso, M.; Vahid, S.; Foot, P.J.S.; Pearce, J.M. Technical pathways for distributed recycling of polymer composites for distributed manufacturing: Windshield wiper blades. Resour. Conserv. Recycl. 2020, 157, 104810. [CrossRef]

49. Meyer, T.K.; Tanikella, N.G.; Reich, M.J.; Pearce, J.M. Potential of distributed recycling from hybrid manufacturing of 3-D printing and injection molding of stamp sand and acrylonitrile styrene acrylate waste composite. Sustain. Mater. Technol. 2020, 25, e00169. [CrossRef]

50. Zander, N.E.; Gillan, M.; Burckhard, Z.; Gardea, F. Recycled polypropylene blends as novel 3D printing materials. Addit. Manuf. 2019, 25, 122-130. [CrossRef]

51. Awaja, F.; Pavel, D. Recycling of PET. Eur. Polym. J. 2005, 41, 1453-1477. [CrossRef]

52. La Mantia, F.P. Polymer Mechanical Recycling: Downcycling or Upcycling? Prog. Rubber Plast. Recycl. Technol. 2004, 20, 11-24. [CrossRef]

53. Polyethylene Terephthalate Production, Price and Market-Plastics Insight. Available online: https://www. plasticsinsight.com/resin-intelligence/resin-prices/polyethylene-terephthalate/ (accessed on 11 May 2020). 
54. Karayannidis, G.P.; Achilias, D.S. Chemical Recycling of Poly(ethylene terephthalate). Macromol. Mater. Eng. 2007, 292, 128-146. [CrossRef]

55. Nace, T. We're Now at A Million Plastic Bottles Per Minute-91\% of Which Are Not Recycled. Available online: https://www.forbes.com/sites/trevornace/2017/07/26/million-plastic-bottles-minute-91not-recycled/ (accessed on 11 May 2020).

56. B-Pet|Bottle PET Filament. Available online: https://bpetfilament.com/ (accessed on 8 May 2020).

57. RE PET 3D|Recycled PET Filament. Available online: https://re-pet3d.com/ (accessed on 8 May 2020).

58. Mosaddek, A.; Kommula, H.K.R.; Gonzalez, F. Design and Testing of a Recycled 3D Printed and Foldable Unmanned Aerial Vehicle for Remote Sensing. In Proceedings of the 2018 International Conference on Unmanned Aircraft Systems (ICUAS), Dallas, TX, USA, 12-15 June 2018; pp. 1207-1216.

59. Refil|The Makers of Recycled Filament|Order Today. Available online: https://www.re-filament.com/ (accessed on 8 May 2020).

60. Zander, N.E.; Gillan, M.; Lambeth, R.H. Recycled polyethylene terephthalate as a new FFF feedstock material. Addit. Manuf. 2018, 21, 174-182. [CrossRef]

61. Woern, A.L.; Byard, D.J.; Oakley, R.B.; Fiedler, M.J.; Snabes, S.L.; Pearce, J.M. Fused Particle Fabrication 3-D Printing: Recycled Materials' Optimization and Mechanical Properties. Materials 2018, 11, 1413. [CrossRef] [PubMed]

62. Alzahrani, M. Modification of Recycled Poly(ethylene terephthalate) for FDM 3D-Printing Applications. Master's Thesis, University of Waterloo, Waterloo, ON, Canada, April 2017.

63. PET Facts|IBWA|Bottled Water. Available online: https://www.bottledwater.org/education/recycling/pet-facts (accessed on 11 May 2020).

64. Parado-Guilford, C. Can Recycled 3D Printing Filament Lead to a Successful Social Venture? Available online: https://blogs.worldbank.org/digital-development/can-recycled-3d-printing-filament-leadsuccessful-social-venture (accessed on 11 May 2020).

65. Techfortrade's Thunderhead PET Filament Extruder Technical Feasibility Study. Available online: http://www.refabdar.org/updates/2016/8/31/techfortrade-thunderhead-pet-filament-extruder-technicalfeasibility-study (accessed on 11 May 2020).

66. Low Speed Open Rotor Scissor Cut Granulators|Shini USA 2016. Available online: https://www.shiniusa.com/ products/granulating-recycling/low-speed-granulators/low-speed-open-rotor-scissor-cut-granulators-2/ (accessed on 11 May 2020).

67. ImageJ. Available online: https://imagej.nih.gov/ij/download.html (accessed on 8 May 2020).

68. Circularity. Available online: https://imagej.nih.gov/ij/plugins/circularity.html (accessed on 13 May 2020).

69. Ultrafuse rPET Natural Blue. Available online: https://www.ultrafusefff.com/product-category/sustainable/ innocircle/ (accessed on 13 May 2020).

70. Haider, A.; Levenspiel, O. Drag coefficient and terminal velocity of spherical and nonspherical particles. Powder Technology 1989, 58, 63-70. [CrossRef]

71. GigabotX OS Cooling System. Available online: https://osf.io/q2bkd/ (accessed on 22 August 2020).

72. Demirel, B.; Yaraş, A.; Elçiçek, H. Crystallization Behavior of PET Materials (PET malzemelerin kristalizasyon davranıș) 2011. Available online: https://www.researchgate.net/profile/Ali_Yaras/publication/290429725_ Crystallization_Behavior_of_PET_Materials/links/56977b7308ae1c427904dc10/Crystallization-Behavior-ofPET-Materials.pdf (accessed on 19 May 2020).

73. Resin Properties Table|PMC. Available online: https://www.pmcplastics.com/materials/pet-resin/ (accessed on 19 May 2020).

74. Overview of Materials for Polyethylene Terephthalate (PET), Unreinforced. Available online: http://www. matweb.com/search/DataSheet.aspx?MatGUID=a696bdcdff6f41dd98f8eec3599eaa20\&ckck=1 (accessed on 13 May 2020).

75. Matias, Á.A.; Lima, M.S.; Pereira, J.; Pereira, P.; Barros, R.; Coelho, J.F.J.; Serra, A.C. Use of recycled polypropylene/poly(ethylene terephthalate) blends to manufacture water pipes: An industrial scale study. Waste Manag. 2020, 101, 250-258. [CrossRef] [PubMed]

76. Wittbrodt, B.; Pearce, J.M. The effects of PLA color on material properties of 3-D printed components. Addit. Manuf. 2015, 8, 110-116. [CrossRef]

77. Tymrak, B.M.; Kreiger, M.; Pearce, J.M. Mechanical properties of components fabricated with open-source 3-D printers under realistic environmental conditions. Mater. Des. 2014, 58, 242-246. [CrossRef] 
78. Pearce, J. Distributed Manufacturing of Open-Source Medical Hardware for Pandemics. J. Manuf. Mater. Process. 2020. [CrossRef]

79. Tino, R.; Moore, R.; Antoline, S.; Ravi, P.; Wake, N.; Ionita, C.N.; Morris, J.M.; Decker, S.J.; Sheikh, A.; Rybicki, F.J.; et al. COVID-19 and the role of 3D printing in medicine. 3D Print. Med. 2020, 6, 11. [CrossRef]

80. Livingston, E.; Desai, A.; Berkwits, M. Sourcing Personal Protective Equipment during the COVID-19 Pandemic. JAMA 2020, 323, 1912-1914. [CrossRef]

81. Ishack, S.; Lipner, S.R. Applications of 3D Printing Technology to Address COVID-19 Related Supply Shortages. Am. J. Med. 2020. [CrossRef]

82. Pearce, J.M. Expanding the Consumer Bill of Rights for material ingredients. Mater. Today 2018, 21, 197-198. [CrossRef]

83. Vidakis, N.; Petousis, M.; Maniadi, A.; Koudoumas, E.; Vairis, A.; Kechagias, J. Sustainable Additive Manufacturing: Mechanical Response of Acrylonitrile-Butadiene-Styrene over Multiple Recycling Processes. Sustainability 2020, 12, 3568. [CrossRef]

84. Cruz, S.A.; Zanin, M. Evaluation and identification of degradative processes in post-consumer recycled high-density polyethylene. Polym. Degrad. Stab. 2003, 80, 31-37. [CrossRef]

85. Santander, P.; Cruz Sanchez, F.A.; Boudaoud, H.; Camargo, M. Closed loop supply chain network for local and distributed plastic recycling for 3D printing: A MILP-based optimization approach. Resour. Conserv. Recycl. 2020, 154, 104531. [CrossRef]

(C) 2020 by the authors. Licensee MDPI, Basel, Switzerland. This article is an open access article distributed under the terms and conditions of the Creative Commons Attribution (CC BY) license (http://creativecommons.org/licenses/by/4.0/). 